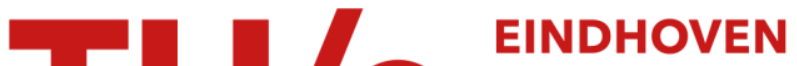

\section{A modified Maxwell-Stefan model for transport through inert membranes : the binary friction model}

\section{Citation for published version (APA):}

Kerkhof, P. J. A. M. (1996). A modified Maxwell-Stefan model for transport through inert membranes : the binary friction model. Chemical Engineering Journal, 64(3), 319-343. https://doi.org/10.1016/S0923-0467(96)03134-X, https://doi.org/10.1016/S0923-0467(96)03121-1

\section{DOI:}

10.1016/S0923-0467(96)03134-X

10.1016/S0923-0467(96)03121-1

\section{Document status and date:}

Published: 01/01/1996

\section{Document Version:}

Publisher's PDF, also known as Version of Record (includes final page, issue and volume numbers)

\section{Please check the document version of this publication:}

- A submitted manuscript is the version of the article upon submission and before peer-review. There can be important differences between the submitted version and the official published version of record. People interested in the research are advised to contact the author for the final version of the publication, or visit the $\mathrm{DOI}$ to the publisher's website.

- The final author version and the galley proof are versions of the publication after peer review.

- The final published version features the final layout of the paper including the volume, issue and page numbers.

Link to publication

\section{General rights}

Copyright and moral rights for the publications made accessible in the public portal are retained by the authors and/or other copyright owners and it is a condition of accessing publications that users recognise and abide by the legal requirements associated with these rights.

- Users may download and print one copy of any publication from the public portal for the purpose of private study or research.

- You may not further distribute the material or use it for any profit-making activity or commercial gain

- You may freely distribute the URL identifying the publication in the public portal.

If the publication is distributed under the terms of Article 25fa of the Dutch Copyright Act, indicated by the "Taverne" license above, please follow below link for the End User Agreement:

www.tue.nl/taverne

Take down policy

If you believe that this document breaches copyright please contact us at:

openaccess@tue.nl

providing details and we will investigate your claim. 


\title{
A modified Maxwell-Stefan model for transport through inert membranes: the binary friction model
}

\author{
Piet J.A.M. Kerkhof * \\ Laboratory for Separation Technology and Transport Phenomena, Faculty of Chemical Engineering and Chemistry, Eindhoven University of Technology, \\ P.O. Box $513,5600 \mathrm{MB}$ Eindhoven, Netherlands
}

Received 12 July 1995; accepted 15 February 1996

\begin{abstract}
This paper focuses mainly on the development of a model for permeation through inert membranes, as encountered in many cases in ultrafiltration and in gas permeation through inert porous plugs. The ultrafiltration model is made up of a boundary layer transport model and a porous membrane model in series, which are connected by an equilibrium relation. The boundary layer model is developed with the Vieth approximation for turbulent diffusivity. For the internal membrane transport, a modification of the Maxwell-Stefan-Lightfoot equation is derived (the binary friction model), which in a natural way includes both interspecies (diffusive) and species-wall forces. Application for the partial separation of PEG-3400 from aqueous solution shows that membrane friction coefficients can simply be estimated from membrane resistance measurements and mixture viscosity data. The only adjustable parameter to be determined is the distribution coefficient between the free solution and the membrane pores. The differences between the Lightfoot approach and the dusty gas model (DGM) are shown to stem from errors in the derivations of the latter, thus invalidating the dusty gas approach in the normal region in which viscous friction effects become important. For gases, the binary friction model is developed to include Knudsen and viscous wall friction terms as well as intermolecular diffusion. It is shown to give excellent coverage of the He-Ar diffusion data of Evans et al. (J. Appl. Phys., 33 (1962) 2682; 34 (1963) 2020), with wall friction coefficients derived directly from Knudsen coefficients and gas viscosity data. The apparent success of the DGM in describing the same phenomena is shown to be caused by the relatively small importance of the wall friction forces at elevated pressures, and by the correct transition to Knudsen flow at low pressures. In addition, it is shown that diffusive slip phenomena in capillaries can be described well by the binary friction model.
\end{abstract}

Keywords:' Binary friction model; Inert membranes; Maxwell-Stefan model; Transport

\section{Introduction}

Ultrafiltration has become a very important unit operation in a variety of industries, the most prominent of which are the food and bioprocess industry. An important factor in many processes is the rejection of various components by the membrane, which generally differs for each component. In addition to the membrane properties and molecular size, the rejection depends on process conditions, such as the circulation velocity, applied transmembrane pressure and feed concentration. In the recent literature, several studies have dealt with the modelling of the transport in and close to the membrane or other porous media [1-14]. In our laboratory too, extensive work has been done by van Oers et al. [1517], who showed that the retention of permeable components could be either enhanced or decreased by the presence of

* Corresponding author. Tel.: 31-40-247 2973; fax: 31-40-244 6104; email: tgtcpk2@chem.tue.nI other components, depending on their nature and process conditions. For the case of ultrafiltration and diafiltration of industrial enzymes, the consequences of the rejection phenomena, as related to process conditions in terms of the required membrane area and processing time, have also been the subject of earlier studies [18-20]. From industrial practice, there is thus a considerable need to have available reliable transport models which require a minimum of adjustable parameters. With respect to the present state of the art, some improvements are considered necessary. The first is the application of a (multicomponent) boundary layer theory to the polarization layer near the membrane. Until now, studies have used a film approximation in which the properties do not depend explicitly on the flow field near the membrane. This implies that effective mass transfer coefficients will be proportional to the molecular (Fickian) diffusion coefficient $D$, and that the film thickness is adjusted to obtain consistency with experimental correlations. Thermodynamic effects and 
component interactions will require more precise modelling of the separate contributions of turbulence and diffusion. The second improvement involves the description of the transport inside the membrane. Here, we explicitly assume the membrane to be heterogeneous. Modelling intramembrane transport has been the subject of considerable study. Two main lines of consideration can be distinguished: the continuum approach and hydrodynamic models. Starting points for most continuum descriptions are the treatments of Lightfoot [1] and the "dusty gas" model of Mason and coworkers [29], extended to the "dusty fluid" model by Krishna [12,13]. The work of Mason, in turn, is based on a statistical mechanics approach. Hydrodynamic models consider the motion of idealized particles of various shapes inside pores of different geometries $[21,22]$. A considerable problem is still the reconciliation of hydrodynamic model parameters with those from thermodynamically based continuum models. Thermodynamic factors play a crucial role in transport in multicomponent ultrafiltration, as demonstrated by van Oers [15], and there is a rigorous base from irreversible thermodynamics for the Maxwell-Stefan-type description. This was shown in earlier work by Mason and coworkers [2-9]; a very clear overview has been given in the recent book of Taylor and Krishna [23], and in the book of Kuiken [24]. Wesselingh and Krishna [25] show a very lucid physical interpretation of many mass transport phenomena.

From these considerations, it follows that the most promising models for ultrafiltration stem from the continuum approach. However, there are a few major problems. One practical problem is the large number of adjustable parameters in the friction coefficients for the solution phase inside the pore and between components and the membrane. Van Oers [15] performed experiments on the transport of PEG3400 in a tubular membrane module, and showed that the results could be described by the Maxwell-Stefan equations along the lines of the dusty gas model (DGM); in addition to separately determined data, five parameters had to be fitted.

A fundamental problem is the choice between the Lightfoot friction model and the DGM. Although Mason et al. showed an algebraic correspondence between the two models, they stated that the $D_{i j}$ values in the Lighfoot model were not molecular diffusion coefficients, but rather "augmented diffusion coefficients", denoted by $E_{i j}$, in which viscous effects were concealed. It was felt that the DGM equations led to certain counterintuitive results in the case of diffusion with a stagnant component and for the permeation of pure liquids. It was thought necessary to try to resolve this matter; therefore, in the first part of this paper, the main lines of derivation of both models are reviewed. It is shown that the addition of a viscous flux to the friction equations, as performed in the DGM, is erroneous, because the viscous friction has already been accounted for in the equations before the addition. This is demonstrated for both the derivation for gases starting from the Zdhanov equation and for liquids starting from the equations of Bearman and Kirkwood [26] and Bearman [27]. It is shown that the statistical mechanical basis also leads to the Lightfoot equation.

Starting from the Lighfoot equation, we develop a relationship between the species-wall friction forces, which enables the species-wall friction coefficients to be determined from bulk mixture viscosity data. Since the resulting equation only contains binary interaction forces (also for species-wall forces), this extended model is called the binary friction model (BFM). We first develop this model for liquid transport. We follow with the application of the boundary layer theory to transport in the polarization layer, and compare the results with the experimental data of van Oers [15].

In addition, we develop the binary friction equations for the transport of gases through porous media, including viscous and diffusive slip phenomena. The results are shown to agree well with the well-known experimental data on $\mathrm{He}-\mathrm{Ar}$ diffusion through graphite of Evans et al. [28,29], and with several results obtained for counterdiffusion in capillaries, such as the Kramers-Kistemaker effect and related phenomena.

\section{Intramembrane transport: classical treatment}

\subsection{The Lightfoot friction model}

It was thought that it would be useful to go through the derivations performed by Lightfoot, since they are spread over various sections in his book [1]; it is shown that his result for free liquid diffusion is unnecessarily complicated and may lead to confusion. The system we consider is a heterogeneous system with an inert membrane, through which a fluid mixture of $n$ components flows. The pore diameter is assumed to be large with respect to the mean free path of the molecules. The fluxes are taken per unit area of the pore and the gradients are taken along the path of the pore; in a later section, we deal with porosity and tortuosity effects separately. Here, we follow the treatment along the lines described by Lightfoot [1] and Taylor and Krishna [23]. First, we regard the classical derivation of the Lightfoot equation for the driving force, which in the first consideration does not contain any reference to a membrane system. Let us denote the number of components in the fluid by $n$; in derivations in which the membrane is included by various workers, we denote this as component $(n+1)$ in summations or $m$ in subscripts. In this paper, we use the tilde symbol for the notation of three-dimensional vectors and operators, e.g. $\tilde{u}$. For vectorial quantities with directions along the pore path, which have been suitably averaged over the pore crosssection, we use the arrow notation, e.g. $\vec{u}$.

We start with the equation for entropy production with the assumption that only scalar products are of importance and that suitable averaging over the membrane pore area is allowed $[2,23]$

$$
T \sigma=-\sum_{i=1}^{n}\left(\frac{1}{M_{i}} \tilde{\nabla}_{T} \mu_{i}-\tilde{F}_{i}\right) \cdot{\tilde{j_{i}}}
$$


in which ${\tilde{J_{i}}}_{i}$ are the mass fluxes with respect to the mass average velocity $\tilde{v}$, defined as

$\tilde{J_{i}} \equiv \rho_{i}\left(\tilde{u_{i}}-\tilde{v}\right)$

$\tilde{F}_{i}$ are the body forces per unit of mass of component $i$, e.g. gravitational, centrifugal or electrical forces.

In the classical treatment, a zero vector is now added to the driving force

$\tilde{F}^{\prime} \equiv \frac{1}{\rho_{\mathrm{t}}} \tilde{\nabla} P+\sum_{i=1}^{n} \omega_{i} \tilde{F}_{i}=\tilde{0}$

"for convenience's sake" as stated by Lightfoot [1]. Eq. (3) is derived from the mechanical equilibrium by Lightfoot [1] and by Taylor and Krishna [23]. This leads to the following equations for entropy production

$T \sigma=-\sum_{i=1}^{n}\left(\frac{1}{M_{i}} \tilde{\nabla}_{T, P} \mu_{i}+\frac{\vec{V}_{i}}{M_{i}} \tilde{\nabla} P-\frac{1}{\rho_{t}} \tilde{\nabla} P-\tilde{F}_{i}+\sum_{j=1}^{n} \omega_{j} \tilde{F}_{j}\right) \cdot \tilde{J}_{i}$

or with

$\sigma=-c_{\mathrm{t}} R \sum_{i=1}^{n} \tilde{d}_{i} \cdot\left(\tilde{u}_{i}-\tilde{v}\right)$

The driving force is defined by

$c_{\mathrm{l}} R T \tilde{d}_{i} \equiv c_{i} \tilde{\nabla}_{T, P} \mu_{i}+\left(c_{i} \bar{V}_{i}-\omega_{i}\right) \tilde{\nabla} P-\rho_{i}\left(\tilde{F}_{i}-\sum_{j=1}^{n} \omega_{j} \tilde{F}_{j}\right)$

Subsequently, the driving force is equated to the friction force by means of

$\tilde{d}_{i}=-\sum_{j=1}^{n} \beta_{i j}\left(\tilde{u}_{i}-\tilde{u}_{j}\right)$

Now, classically, the friction terms are written as

$\beta_{i j}=\frac{x_{i} x_{j}}{D_{i j}}$

which then leads to the well-known equation

$$
\begin{aligned}
& \frac{x_{i}}{R T} \tilde{\nabla}_{T, P} \mu_{i}+\frac{\left(\phi_{i}-\omega_{i}\right)}{c_{\mathrm{t}} R T} \tilde{\nabla} P-\frac{\rho_{i}}{c_{\mathrm{t}} R T}\left(\tilde{F}_{i}-\sum_{j=1}^{n} \omega_{j} \tilde{F}_{j}\right) \\
& \quad=\sum_{j=1}^{n} \frac{x_{i} \tilde{N}_{j}-x_{j} \tilde{N}_{i}}{c_{\mathrm{t}} D_{i j}}
\end{aligned}
$$

and, as shown by Lightfoot [1] and Taylor and Krishna [23], for the Maxwell-Stefan diffusion coefficients

$\bigoplus_{i j}=\boxplus_{j i}$

Up to this point, the treatment of Lightfoot considers only fluid components, and does not specify the dimensionality of the vectors considered; therefore, up to Eq. (9), the vectors may also be considered to be local three-dimensional vectors.

The next step in Lightfoot's development is the introduction of the membrane as a separate component, denoted by $m$; according to his derivation, because of the "clamping force" to keep the membrane in place, the mechanical equilibrium leads to

$$
\vec{F}_{i}-\sum_{\substack{j=1 \\ j \neq m}}^{n+1} \omega_{j} \vec{F}_{j}=\vec{F}_{\mathrm{el}}+\vec{F}_{\text {grav }}-\frac{1}{\rho} \vec{\nabla} P
$$

in which the summation is now carried out over the $n$ components making up the mixture. Without explicitly mentioning this, Lightfoot changes to pore-averaged fluxes and operators, and tacitly assumes that all local equations can also be written for the averaged values. Eq. (11) leads to the driving force expression

$c_{\mathrm{t}} R T \vec{d}_{i} \equiv c_{i} \vec{\nabla}_{T, P} \mu_{i}+c_{i} \overrightarrow{V_{i}} \vec{\nabla} P-\rho_{i} \vec{F}_{i}$

In the final step, the friction with the membrane is written separately and the membrane velocity is taken to be equal to zero; the multicomponent version of Lightfoot's binary solution equation then becomes

$$
\frac{x_{i}}{R T} \vec{\nabla}_{T, P} \mu_{i}+\frac{\phi_{i}}{c_{\mathrm{l}} R T} \vec{\nabla} P-\frac{\rho_{i}}{c_{\mathrm{t}} R T} \vec{F}_{i}=\sum_{j=1}^{n} R_{i j}\left(x_{i} \vec{N}_{j}-x_{j} \vec{N}_{i}\right)-r_{\mathrm{im}} \vec{N}_{i}
$$

with the "resistance factors" given by

$R_{i j}=\frac{1}{c_{\mathrm{t}} D_{i j}}$

$r_{i \mathrm{~m}}=\frac{x_{\mathrm{m}}}{c_{\mathrm{i}} D_{i \mathrm{~m}}}=\frac{\beta_{i \mathrm{~m}}}{c_{i}}$

thus avoiding the introduction of the membrane molecular mass.

The foregoing derivation may be considerably simplified by starting directly with the fluid system as such, without including the membrane as a possible component. Thus the membrane is considered as an external body which does not influence the local thermodynamic and transport properties of the fluid in relation to intensive variables, but which exerts a friction force on the fluid species. Thus, in the following, $n$ is again the number of components in the solution and does not include the membrane.

We start again with Eq. (1), without including the zero vector (Eq. (3)), and obtain for the $n$-component fluid system

$$
\begin{aligned}
T \sigma & =-\sum_{i=1}^{n}\left(\frac{1}{M_{i}} \tilde{\nabla}_{T, P} \mu_{i}+\frac{\bar{V}_{i}}{M_{i}} \tilde{\nabla} P-\tilde{F}_{i}\right) \cdot \tilde{J}_{i} \\
& =-\sum_{i=1}^{n}\left(c_{i} \tilde{\nabla}_{T, P} \mu_{i}+c_{i} \bar{V}_{i} \tilde{\nabla} P-\rho_{i} \tilde{F}_{i}\right) \cdot\left(\tilde{u}_{i}-\tilde{v}\right)
\end{aligned}
$$

and so we find directly the driving force for motion on species $i$ per unit volume of mixture as

$c_{t} R T \tilde{e}_{i}=c_{i} \tilde{\nabla}_{T, P} \mu_{i}+c_{i} \bar{V}_{i} \tilde{\nabla} P-\rho_{i} \tilde{F}_{i}$ 
Thus we find for the free solution

$\frac{x_{i}}{R T} \tilde{\nabla}_{T, P} \mu_{i}+\frac{\phi_{i}}{c_{t} R T} \tilde{\nabla} P-\frac{\rho_{i}}{c_{i} R T} \tilde{F}_{i}=\sum_{j=1}^{n} \frac{x_{i} \tilde{N}_{j}-x_{j} \tilde{N}_{i}}{c_{t} D_{i j}}$

Although this result may seem trivial regarding the definition in Eq. (3), it is my experience that students new in the field tend to start with Eq. (9) without regarding Eq. (3), and in the case of absent external forces they end up with the wrong equation because only the third term in Eq. (9) is left out.

With the assumption that with pore-averaged quantities the equations remain intact, it seems plausible for the transport in the membrane to add a friction force with the membrane to the averaged intermolecular friction terms; therefore we obtain for the transport in the pores

$\frac{x_{i}}{R T} \vec{\nabla}_{T, P} \mu_{i}+\frac{\phi_{i}}{c_{\mathrm{t}} R T} \vec{\nabla} P-\frac{\rho_{i}}{c_{\mathrm{t}} R T} \vec{F}_{i}=\sum_{j=1}^{n} \frac{x_{i} \vec{N}_{j}-x_{j} \vec{N}_{i}}{c_{\mathrm{t}} \theta_{i j}}-r_{i \mathrm{~m}^{2}} \vec{N}_{i}$

identical to the Lightfoot result (Eq. (13)). Lightfoot showed that without loss of generality a thermal diffusion term may be added to the friction equation.

Although convenient from the engineering point of view, the use of pore-averaged fluxes and driving forces is by no means self-evident. Further study is desirable to determine whether and under which assumptions we can obtain the poreaveraged equations, starting with the averaging of the entropy production equation (Eq. (4)).

\subsection{Treatment of Mason et al: the DGM}

In a series of papers, Mason and coworkers have treated the equations for transport in porous systems and applied these to membranes, first for gases and later for liquids $[2,3,8,30]$. An extensive review of this work can also be found in Cunningham and Williams [31].

The basic physical assumption in the DGM is that we can split up the flux of a component into a diffusive and a viscous part

$N_{i}=N_{i}^{\mathrm{D}}+x_{i} N_{\text {visc }}$

in which the viscous part represents the motion of the component due to the flow of the mixture as a whole, and was initially identified as

$\tilde{N}_{i, \text { visc }}=-\frac{x_{i} B_{0} c_{t}}{\eta} \tilde{\nabla} P$

where $B_{\mathrm{o}}$ is the permeability of the porous medium, which for cylindrical pores with radius $r_{\mathrm{o}}$ is equal to $B_{0}=r_{\mathrm{o}}^{2} / 8$.

In addition, it is stated that this is a consequence of the theoretical development from statistical mechanics in which, in the first-order approximation of the Boltzmann integral, no viscous terms remain in the diffusion equation and in the momentum balance no diffusion coefficients occur $[8,30]$. In their derivations for gases, Mason et al. picture the wall as consisting of giant dust molecules uniformly distributed in space, which are held motionless by a clamping force. They obtain an expression which, for the isothermal case and in the absence of external forces, may be written as

$\frac{1}{P_{\mathrm{t}}} \vec{\nabla}_{T} P_{i}=\sum_{j=1}^{n} \frac{x_{i} \vec{N}_{j}-x_{j} \vec{N}_{i}}{c_{\mathrm{t}} D^{\prime}{ }_{i j}}-\frac{c_{\mathrm{d}} \vec{N}_{i}}{c_{\mathrm{t}}^{2} D^{\prime}{ }_{i \mathrm{~d}}}=\sum_{j=1}^{n} \frac{x_{i} \vec{N}_{j}-x_{j} \vec{N}_{i}}{c_{\mathrm{t}} D^{\prime}{ }_{i j}}-\frac{\vec{N}_{i}}{c_{\mathrm{t}} D^{\prime}{ }_{\mathrm{im}}}$

In this equation, the prime on the diffusion coefficients denotes the inclusion of the second-order corrections in which the dust (d) is not included in the summation. It is stated that the fluxes appearing in Eq. (20) are only the diffusive fluxes, and that for completeness the viscous fluxes should be added (see their Eq. (63)), which may be written as

$\frac{1}{P_{\mathrm{t}}} \vec{\nabla}_{T} P_{i}=\sum_{j=1}^{n} \frac{x_{i} \vec{N}_{j}-x_{j} \vec{N}_{i}}{c_{\mathrm{t}} D^{\prime}{ }_{i j}}-\frac{\vec{N}_{i}}{c_{\mathrm{t}} D^{\prime}{ }_{i \mathrm{~m}}}+\frac{x_{i} \vec{N}_{\mathrm{visc}}}{c_{\mathrm{t}} D^{\prime}{ }_{i \mathrm{~m}}}$

and

$\vec{N}_{\text {visc }}=-\frac{c_{\mathrm{t}} B_{\mathrm{o}}}{\eta} \vec{\nabla} P$

For the flux of a single component, the Maxwell-Stefan term equals zero and Eq. (21) becomes

$\vec{N}_{i}=-\frac{1}{R T}\left(D^{\prime}{ }_{i \mathrm{~m}}+\frac{B_{\mathrm{o}} P_{\mathrm{t}}}{h}\right) \vec{\nabla}_{\mathrm{T}} P_{\mathrm{t}}$

which corresponds to the viscous slip equation when $D_{i}^{K}=$ $D^{\prime}{ }_{i m}$, as stated by Mason et al. It is understood here that, in $D_{\mathrm{i}}^{\mathrm{K}}$, the Knudsen pressure effect may be included $[8,31]$.

For liquids, we first consider the final result as developed by Mason and del Castillo [2]. In the present notation, again in the absence of external forces on the fluid molecules and for isothermal conditions

$\frac{x_{i}}{R T}\left(\vec{\nabla}_{T, P} \mu_{i}+\bar{V}_{i} \vec{\nabla} P\right)=\sum_{j=1}^{n} \frac{x_{i} \vec{N}_{j}-x_{j} \vec{N}_{i}}{c_{\imath} D_{i j}}-\frac{\vec{N}_{i}}{c_{i} D_{i \mathrm{~m}}}-\frac{\alpha_{i}^{\prime} B_{0} x_{i}}{\eta D_{i \mathrm{~m}}} P$

We have now written the Maxwell-Stefan coefficients as $D_{i j}$ rather than $\nexists_{i j}$ in view of the fact that they are not identical to those in the Lightfoot equations (Eq. (13) and Eq. (17)), as follows from Mason's treatment which will be discussed shortly. The difference from the Lightfoot equation (Eq. (13)) again lies in the last term, which stands for the so-called "viscous flow" contribution, and which includes the permeability $B_{\circ}$ of the membrane, the viscosity $\eta$ of the whole fluid and $\alpha_{i}^{\prime}$, which are species-dependent coefficients for the effect of the viscous flow. Originally, $\alpha_{i}^{\prime}$ were not included in the development, but were introduced later in a discussion about semipermeability in order to be able to describe "separative viscous flow" [2]. In later papers, Eq. (24) is stated to have been obtained from the "statistical mechanical treatment", and in a number of papers it is stressed that friction-type equations, such as the Lightfoot equation, obscure the separate effects of diffusion and viscous 
flow $[2,9]$. Mason and del Castillo's version of the frictionbased equation in the absence of external forces reads

$\frac{x_{i}}{R T}\left(\vec{\nabla}_{T, P} \mu_{i}+\bar{V}_{i} \vec{\nabla} P\right)=\sum_{j=1}^{n} \frac{x_{i} \vec{N}_{j}-x_{j} \vec{N}_{i}}{c_{t} E_{i j}}-\frac{\vec{N}_{i}}{c_{\mathrm{t}} E_{i \mathrm{~m}}}$

They designate $E_{i j}$ as "augmented diffusion coefficients", and show that an algebraic relationship exists in the following form

$E_{i \mathrm{~m}}=D_{i \mathrm{~m}}(1+\beta)\left[1+\beta\left(1-\frac{\alpha_{i}^{\prime} \bar{D}_{\mathrm{m}}}{\bar{D}_{\mathrm{m}}^{\circ}}\right)\right]^{-1}$

$E_{i j}=\left[\frac{1}{D_{i j}}+\frac{\alpha^{\prime} \vec{D}_{\mathrm{m}}}{D_{i \mathrm{~m}} D_{j \mathrm{~m}}}\left(\frac{\beta}{1+\beta}\right)\right]^{-1}$

$\beta \equiv \frac{c_{\mathrm{t}} B_{\mathrm{o}} R T}{\eta \bar{D}_{\mathrm{m}}}$

$\frac{1}{\bar{D}_{\mathrm{m}}} \equiv \sum_{j=1}^{n-1} \frac{\alpha_{j}^{\prime} x_{j}}{D_{j \mathrm{~m}}}$

$\frac{1}{\bar{D}_{\mathrm{m}}^{\mathrm{o}}} \equiv \sum_{j=1}^{n-1} \frac{x_{j}}{D_{j \mathrm{~m}}}$

As stated by Mason and Lonsdale [9], the introduction of $\alpha^{\prime}{ }_{i}$ leads to breaking of the Onsager symmetry for the poreaveraged transport coefficients; they attribute this to the difference between the pore-averaged quantities and local quantities for which the Onsager reciprocity relations hold, and state that the integration is the cause of symmetry breaking.

From the statements of Mason and coworkers in their various papers, it is clear that they consider that the dusty gastype model is superior to friction-type models, and they ascribe the success of the application of friction-type models to the fact that there is an algebraic equivalence.

\subsection{Some problems with the DGM equations, and proof of their incorrectness}

In my view, the DGM equations have some counterintuitive consequences. Let us first consider a problem in gas transport, the transport of water vapour in a capillary tube, in which at $z=0$ water evaporates and at $z=L$ it leaves the tube into the surrounding air ("Stefan tube"). By choosing a sufficiently small capillary radius and a high enough water temperature, we have a reasonable pressure difference between the evaporating front and the air. (For a capillary of $10^{-5} \mathrm{~m}$ diameter and $0.5 \mathrm{~m}$ length, and a water temperature of $100.9^{\circ} \mathrm{C}$, the overpressure will be approximately 0.047 bar under atmospheric external conditions.) It is generally assumed that we can consider the air in the tube as stagnant. The pressure gradient in the air will follow from the DGM equations for $\vec{N}_{\mathrm{a}}=\overrightarrow{0}$ as

$\frac{1}{P_{\mathrm{t}}} \vec{\nabla}_{T} P_{\mathrm{a}}=\frac{x_{\mathrm{a}} \vec{N}_{\mathrm{w}}}{c_{\mathrm{t}} D^{\prime}{ }_{\mathrm{aw}}}-\frac{x_{\mathrm{a}} B_{\mathrm{o}}}{\eta D^{\prime}{ }_{\mathrm{am}}} \vec{\nabla} P_{\mathrm{t}}$
Since the air is stagnant, we expect that the air partial pressure gradient will be solely due to intermolecular collisions with the moving water vapour, as reflected in the first term on the right-hand side. The viscous term, however, also implies a contribution of viscous friction between the air and the wall, which is against physical intuition. It is noted that in the first equation of the DGM, Eq. (20), before the addition of the viscous flux term, this effect will not be present.

The second problem lies in the area of liquids. As derived previously [3], a consequence of the Mason model is that, for the flow of a single component, e.g. s, in the absence of electrical and gravitational forces, etc., we obtain

$\vec{N}_{\mathrm{s}}=-\left(\frac{D_{\mathrm{sm}}}{R T}+\frac{c_{\mathrm{t}} \alpha_{\mathrm{s}}{ }^{\prime} B_{\mathrm{o}}}{\eta_{\mathrm{s}}}\right) \vec{\nabla} P$

or, as given in the initial work [3]

$\vec{N}_{\mathrm{s}}=-\left(\frac{D_{\mathrm{sm}}}{R T}+\frac{c_{\mathrm{t}} B_{\mathrm{o}}}{\eta_{\mathrm{s}}}\right) \vec{\nabla} P$

This means that, for a pure liquid, there will be two terms describing the friction with the wall. It is generally accepted that, for Newtonian liquids, no slip occurs at the wall in the sense of the rarefied gas phenomena, which give rise to the Maxwell and Kramers and Kistemaker effects. These effects are caused by different molecular velocity distributions between gas molecules returning from the wall and those in the bulk, and are manifested only when there are insufficient intermolecular collisions near the wall [31-33]. Thus, for pure liquids, we would expect a term corresponding to Darcy's law

$\vec{N}_{\mathrm{s}}=-\frac{c_{\mathrm{c}} B_{\mathrm{o}}}{\eta_{\mathrm{s}}} \vec{\nabla} P$

This is also a direct consequence of the definition of the viscous flux itself in Eq. (22). Thus the addition of the viscous flux leads to a contradiction within the model itself.

In order to trace the apparent contradictions, we first inspect the derivations of the DGM for gases as presented in Ref. [30]. Mason et al. start from the Zdhanov result for the thirteen-moment approximation for the solution of the Boltzmann equation, and consider a one-dimensional form, their eq. (1), which for the isothermal situation may be written as

$$
\begin{aligned}
\sum_{j=1}^{n} \frac{x_{j} \tilde{N}_{i}-x_{i} \tilde{N}_{j}}{c_{\mathrm{t}} D^{\prime}{ }_{i j}}= & -\frac{1}{P_{\mathrm{t}}}\left(\frac{\partial P_{i}}{\partial z}-\omega_{i} \frac{\partial P}{\partial z}\right)+\frac{1}{R T}\left(x_{i} \tilde{F}_{i}^{\prime}-\omega_{i} \sum_{j=1}^{n} x_{j} \tilde{F}_{j}^{\prime}{ }_{j}\right) \\
& +\frac{2}{P_{\mathrm{t}}}\left(\eta_{i}+W_{i}-\omega_{i}\right)\left(\frac{\partial^{2} \tilde{v}_{z}}{\partial x^{2}}+\frac{\partial^{2} \tilde{v}_{z}}{\partial y^{2}}+\frac{\partial^{2} \tilde{v}_{z}}{\partial z^{2}}\right)
\end{aligned}
$$

in which $\eta_{i}$ are the partial viscosities and $W_{i}$ are the secondorder viscosity corrections. In $D_{i j}^{\prime}$, the second-order corrections $\Delta_{i j}$ are implied. Eq. (34) only considers the gaseous components. In this equation, $\tilde{v}_{z}$ is the $z$ component of the mass average velocity

$\tilde{v}_{z}=\frac{1}{\rho_{j=1}} \sum_{j=1}^{n} \tilde{N}_{i}$ 
The tilde symbol is used again to indicate three-dimensional vectors. In the following, we assume no external forces on the gas constituents. Although Mason et al. kept the forces in the equations, they finally assumed that they were zero. To leave them out will not change the arguments, but keeps the derivations simpler.

Mason includes the momentum balance for viscous flow

$2 \eta\left(\frac{\partial^{2} \tilde{v}_{z}}{\partial x^{2}}+\frac{\partial^{2} \tilde{v}_{z}}{\partial y^{2}}+\frac{\partial^{2} \tilde{v}_{z}}{\partial z^{2}}\right)=\frac{\partial P_{t}}{\partial z}$

In Eq. (36), $\eta$ is the first-order approximation of the mixture viscosity, and $\sum_{j=1}^{n} \eta_{i}=\eta$.

It is not clear where the factor two comes from; it conflicts with the derivations of Bird et al. [34]; however, it does not influence the results. Mason et al. substitute Eq. (36) into Eq. (34) to remove the divergence term to obtain the equivalent of

$\sum_{j=1}^{n} \frac{x_{j} \bar{N}_{i}-x_{i} \tilde{N}_{j}}{c_{\mathrm{t}} D^{\prime}{ }_{i j}}=-\frac{1}{P_{\mathrm{t}}}\left(\frac{\partial P_{i}}{\partial z}-\frac{\eta_{i}+W_{i}}{\eta} \frac{\partial P_{\mathrm{t}}}{\partial z}\right)$

The next statement is that the fluxes involved in Eq. (37) are the diffusive fluxes, and that the total flux of component $i$ is given by

$N_{i}=N_{i}^{\mathrm{D}}+x_{i} N_{\text {visc }}$

and no explicit averaging is applied over the pore crosssection, but apparently this is done implicitly.

Now the dust particles are added as a component (d) which is held in place by a clamping force. For the momentum exchange between $i$ and the dust particles, a term is added on both sides, leading to

$$
\begin{aligned}
& \sum_{j=1}^{n} \frac{x_{j} N_{i}^{\mathrm{D}}-x_{i} N_{j}^{\mathrm{D}}}{c_{\mathrm{t}} D^{\prime}{ }_{i j}}+\frac{x_{\mathrm{d}} N_{i}^{\mathrm{D}}}{c_{\mathrm{t}} D^{\prime}{ }_{i \mathrm{~d}}} \\
& =-\frac{1}{P_{\mathrm{t}}}\left(\frac{\partial P_{i}}{\partial z}-\frac{\eta_{i}+W_{i}}{\eta} \frac{\partial P_{\mathrm{t}}}{\partial z}\right)-\frac{\eta_{i}+W_{i}}{\eta} \frac{1}{P_{\mathrm{t}}} c_{\mathrm{d}} F^{\prime}{ }_{\mathrm{d}}
\end{aligned}
$$

For the clamping force, a mechanical balance is made which states that the total force on the dust particles equals the total pressure gradient

$c_{\mathrm{d}} F^{\prime}{ }_{\mathrm{d}}=\frac{\partial P_{\mathrm{t}}}{\partial z}$

thus leading to

$\sum_{j=1}^{n} \frac{x_{j} \tilde{N}_{i}-x_{i} \tilde{N}_{j}}{c_{\mathrm{t}} D^{\prime}{ }_{i j}}+\frac{x_{\mathrm{d}} \tilde{N}_{i}}{c_{\mathrm{t}} D^{\prime}{ }_{i \mathrm{~d}}}=-\frac{1}{P_{\mathrm{t}}}\left(\frac{\partial P_{i}}{\partial z}\right)$

For the viscous flux, they then write by consideration of the flow around spheres

$N_{\text {visc }}=-\frac{c_{\mathrm{t}}}{\eta R_{\mathrm{o}}} \frac{\partial P}{\partial z}$

in which $R_{\mathrm{o}}$ is a geometry factor; they estimate $D^{\prime}{ }_{i \mathrm{~d}}$ from kinetic gas theory, in which the collision diameter is made proportional to the diameter of the dust particles. Many other parameters are related to fundamental statistical mechanics quantities, and with those relations it is stated that: "This completes the physics of the problem...", and subsequently a number of special cases are considered. In the first case, that of the flow of a pure gas, $D^{\prime}$ id is identified with the Knudsen flow coefficient, and the addition of the viscous flux results in the well-known slip flow equation

$N=-\frac{1}{R T}\left(D^{\mathrm{K}}+\frac{B_{\mathrm{o}} P}{\eta}\right) \frac{\mathrm{d} P}{\mathrm{~d} z}$

No real connection is made between $D^{\mathrm{K}}$ and the earlier kinetic estimate of $D^{\prime}{ }_{i \mathrm{~d}}$.

The error in the foregoing can be demonstrated as follows. In the starting equation, the stress tensor is clearly based on the assumption that viscous momentum transfer occurs; in addition, the balance in Eq. (36) is based on this assumption. If, hypothetically, we try to modify Eq. (34) for the rarefied situation in which no viscous momentum transfer takes place, this will also mean that intermolecular collisions will no longer occur, and we will obtain zero on both sides. The description of Knudsen flow is directly related to the geometrical constraints, and thus cannot be considered in an unconstrained medium; also, slip flow phenomena are related to the presence of a wall. Eq. (34) in itself cannot be solved for the flux, since it is invariant to a linear coordinate transformation. If we consider the porous material as the fixed coordinate system, we can only find the fluxes in Eq. (34) if we connect $\tilde{v}_{z}$ to our system, which can be done by integration over a representative cross-section of the porous system, and the application of a boundary condition. For sufficiently low Knudsen numbers, we would use a no-slip boundary condition; for slip flow conditions, we might assume an effective velocity at the wall, $v_{w}$, as described by Maxwell [35] for single components and by Kramers and Kistemaker [33] for binary mixtures (see also Ref. [31]).

Assuming low Re numbers, we may perform the integration over a cylindrical pore cross-section or, as performed by Mason et al., over a number of spheres, and subsequently may obtain the pore-averaged mass-averaged velocity in the form

$\vec{v}_{z}=-\frac{B_{\mathrm{o}}}{\eta} \frac{\mathrm{d} P_{\mathrm{t}}}{\mathrm{d} z}+v_{\mathrm{w}}$

Assuming uniform radial concentrations, we can then first integrate Eq. (37) to obtain pore-averaged fluxes, and substitute Eq. (43) for the pressure gradient. Since no introduction of slip flow was made in the derivation of Mason et al., we will for the moment take $v_{\mathrm{w}}=0$. This leads to

$\sum_{j=1}^{n} \frac{x_{j} \vec{N}_{i}-x_{i} \vec{N}_{j}}{c_{\mathrm{t}} D^{\prime}{ }_{i j}}=-\frac{1}{P_{\mathrm{t}}} \frac{\mathrm{d} P_{i}}{\mathrm{~d} z}+\frac{\eta_{i}+W_{i}}{B_{0} P_{\mathrm{t}}} \vec{v}_{z}$

Since $\vec{v}_{z}=(1 / \rho) \sum_{j=1}^{n} \vec{N}_{j} M_{j}$, we can formally translate Eq. (44) into a matrix equation

$[\mathrm{Q}](N)=-(\nabla P)$ 
and solve the equation for the fluxes. This will not be done here, but the basic point has been demonstrated that the set of equations in itself is complete and contains viscous forces, which can only be expressed in averaged fluxes after integration and application of boundary conditions. The steps taken by Mason et al. in Eqs. (38)-(40) merely identify $D_{i \mathrm{~d}}^{\prime}$ by

$\frac{x_{\mathrm{d}} \vec{N}_{i}}{c_{\mathrm{t}} D^{\prime}{ }_{i \mathrm{~d}}}=\frac{\eta_{i}+W_{i}}{B_{\mathrm{o}} P_{\mathrm{t}}} \vec{v}_{z}$

For a single-component (s) flow, it follows with the implied definition of $D^{\prime}{ }_{\mathrm{sm}}=D^{\prime}{ }_{\mathrm{sd}} / x_{\mathrm{d}}$ in Eq. (20) that

$D^{\prime}{ }_{\mathrm{sm}}=\frac{B_{\mathrm{o}} P_{\mathrm{L}}}{\eta}$

Therefore the solution starting from the Zdhanov equations is really based on the assumption of viscous flow, and the results are complete without the addition of an extra term. Instead of describing the slip flow in terms of the Knudsen coefficient as stated by Mason, $D^{\prime}{ }_{i \mathrm{~m}}$ are just the contributions of the viscous stresses. It is clear from Eq. (47) and Eq. (23) that the addition of the viscous flow term by Mason et al. leads to a double counting of the viscous flow and thus is definitely incorrect.

It can therefore be concluded that the final result of the DGM equations is incorrect because of the addition of the viscous flux in the final steps, and that a correct answer in the absence of slip is given by Eq. (44). The introduction of the slip velocity as an approximation is easily seen to give

$\sum_{j=1}^{n} \frac{x_{j} \vec{N}_{i}-x_{i} \vec{N}_{j}}{c_{\mathrm{t}} D_{i j}^{\prime}}=-\frac{1}{P_{\mathrm{t}}} \frac{\mathrm{d} P_{i}}{\mathrm{~d} z}+\frac{\eta_{i}+W_{i}}{B_{\mathrm{o}} P_{\mathrm{t}}}\left(\vec{v}_{z}-v_{\mathrm{w}}\right)$

in which, for a single gas, $v_{w}$ can be expressed in terms of the Knudsen or Maxwell slip relations. Thus Mason et al. should not have added a viscous term to Eq. (44), which already contains the viscous forces, but a Knudsen (slip) term, since that effect was not included in the Zdhanov equation or in their formulation of the pressure drop (Eq. (41)).

The probable cause of the erroneous steps in the derivations of Mason et al. is twofold. Firstly, there is the additivity of the two terms in the slip flow of a single gas. Secondly, there is the confusion caused by the use of terminology. In the statistical mechanics approximations, all velocities are considered with respect to the mass-averaged velocity, and so we have the elementary relation

$\tilde{N}_{i}=\tilde{J}_{i}+c_{i} \tilde{v}$

in which $\tilde{J}_{i}$ is the (molar) flux with respect to the massaveraged velocity $\tilde{v}$. It is an a priori definition of Mason and others that $\tilde{J}_{i}$ is a "diffusive flux", and also that $c_{i} \tilde{v}$ is part of a non-segregative flux, which is termed "viscous". This does not alter the fact that the mathematical treatments of statistical mechanics lead to approximations for $\tilde{J}_{i}$ in relation to $\tilde{v}$. For a binary isothermal system without velocity gradients, these solutions lead to the well-known equation

$\tilde{N}_{1}=-D_{12} \rho \tilde{\nabla} x_{1}+N_{1} x_{1}$
Although often used, this should be regarded as a special case; as follows from the foregoing discussion, things change once velocity gradients are present. It is felt that the ample use of arbitrary kinds of fluxes obscures matters rather than elucidates them. This is especially so in the introductory chapter of Cunningham and Williams [31], in which a large number of different fluxes are defined in relation to various physical phenomena, but a confusion of total and viscous flux occurs. Through these definitions, plausible associations are made and placed into equation form, e.g. the viscous flux, and in other equations the fluxes $\tilde{J}_{i}$ are just stated to be diffusive (incomplete?) and additions are made, thereby violating the rigorous nature of the statistical mechanics derivations.

In the foregoing discussion, the inconsistency in the derivation of the DGM for gases was demonstrated. For the case of liquids, Mason and Viehland [3] used another derivation, which will now be discussed. The basic theoretical starting point in their work is the statistical mechanical work of Bearman [27] and Bearman and Kirkwood [26]. Before going into details, one of the results of the general Bearman and Kirkwood treatment is that, for a mixture, the Navier-Stokes equation is obtained (their Eq. (5.14)) by summing the equations of motion over all components

$\frac{\partial}{\partial t}(\rho \tilde{u})=-\tilde{\nabla} \dot{P}+\eta \nabla^{2} \tilde{u}+\left(s+\frac{1}{3} \eta\right) \tilde{\nabla} \tilde{\nabla} \cdot \tilde{u}+c_{t} \tilde{F}^{\prime}$

Again we assume that we may neglect the body forces and have an isothermal situation; this simplifies the arguments, but again does not change the conclusions. For a liquid with constant density $\tilde{\nabla} \cdot \tilde{u}=0$, from the continuity equation [34] and in the absence of acceleration, Eq. (51) reduces to

$-\tilde{\nabla} P+\eta \nabla^{2} \tilde{u}=\tilde{0}$

For a single component, the statistical mechanics derivations thus lead to the viscous flow of this component. For the low $\mathrm{Re}$ and Mach numbers in membrane transport, as well as in most other porous systems outside the Knudsen region, the integration of the Navier-Stokes equation leads directly to Darcy's law

$\vec{N}_{\mathrm{s}}=-\frac{c_{\mathrm{t}} B_{\mathrm{o}}}{\eta_{\mathrm{s}}} \vec{\nabla} P$

Here the inclusion of porosity and tortuosity effects has been regarded as classical.

Thus there is the same contradiction for liquids as for gases between the outcome of the original statistical mechanics equations applied to a single component in the absence of external forces and the results of Mason and Viehland in Eq. (32). This appears to stem from the following. In the derivation of Mason and Viehland [3], Bearman's result for the component equation of motion appears, which isothermally reads

$-\tilde{J}_{i} \equiv-c_{i}\left(\tilde{u_{i}}-\tilde{u}\right)=\sum_{j=1}^{n^{\prime}} \Omega_{i j} \tilde{Z}_{j}$ 
where in the absence of body forces

$\tilde{Z}_{j}=\tilde{\nabla}_{T} \mu_{j}+\tilde{B}_{j} / c_{j}$

and

$\tilde{B}_{j}=\frac{\partial}{\partial t}\left(\rho_{j} \tilde{u}_{j}\right)+\tilde{\nabla} \cdot\left(\rho_{j} \tilde{u} \tilde{u}\right)-\eta_{j} \nabla^{2} \tilde{u}-\left(\zeta_{j}+\frac{1}{3} \eta_{j}\right) \tilde{\nabla} \tilde{\nabla} \cdot \tilde{u}$

Thus the force $\tilde{Z}_{j}$ is made up of the gradient in the chemical potential and the momentum change rate due to inertial effects and viscous shear.

Eq. (53) is then transformed into

$\sum_{j=1}^{n^{\prime}} \frac{x_{i} x_{j}}{D_{i j}}\left(\tilde{u}_{i}-\tilde{u_{j}}\right)=-\frac{x_{i}}{R T}\left(\tilde{\nabla}_{T} \mu_{i}\right)-\frac{\tilde{B}_{i}}{c_{\mathrm{t}} R T}$

in which we have used molar quantities. The membrane is taken as one component with zero velocity, resulting in

$\sum_{j=1}^{n^{\prime}} \frac{x_{i} x_{j}}{D_{i j}}\left(\tilde{u}_{i}-\tilde{u}_{j}\right)+\frac{x_{i}}{D_{i \mathrm{~m}}} \tilde{u}_{i}=-\frac{x_{i}}{R T}\left(\tilde{\nabla}_{T} \mu_{i}\right)-\frac{\tilde{B}_{i}}{c_{\mathrm{t}} R T}$

As shown by Mason and Viehland [3], summing up the forces $\tilde{Z}_{j}$ over all components leads to

$\sum_{j=1}^{n} c_{j} \tilde{Z}_{j}=\tilde{0}$

and thus

$\tilde{\nabla} P+\sum_{j=1}^{n} \tilde{B}_{j}=\tilde{0}$

or

$\sum_{j=1}^{n} \tilde{B}_{j}=-\tilde{\nabla} P$

They then take the "viscous component of the forced flow" $\vec{u}_{\text {visc }}$ proportional to $\sum_{j=1}^{n} \tilde{B}_{j}$ and, using Eq. (59), state that integration over the whole fluid across a proper cross-section will always lead to

$\vec{u}_{\text {visc }}=-\frac{B_{\mathrm{o}}}{\eta}(\vec{\nabla} P)$

In addition, they take the pore-area integrated $\vec{B}_{j}$ proportional to $x_{j} \vec{u}_{\mathrm{visc}}$. In integrating the equations over the membrane cross-section, we have changed the vectorial notation from $\tilde{u}, \tilde{\nabla}$, etc. to the symbols $\vec{u}, \vec{\nabla}$, etc. to represent one-dimensional vectorial quantities, a change in notation not made by Mason and Viehland.

Subsequently, Mason and Viehland [3] considered the special isothermal case of a single pure species "under conditions where the diffusive flow is negligible compared to the viscous flow"; they assumed that, for this case, the force term $\vec{\nabla}_{T} \mu_{\mathrm{s}}$, described by them as "the term which drives diffusion", "can be dropped", thus obtaining

$\frac{\vec{u}_{\mathrm{visc}}}{D_{\mathrm{sm}}}=\frac{\vec{B}_{\mathrm{s}}}{c_{\mathrm{t}} R T}$
In Eq. (61), we have added the index s. Mason and Viehland then proceeded to find the expression for $\vec{B}_{j}$

$\vec{B}_{j}=-\frac{x_{j} c_{\mathrm{t}} R T}{D_{j \mathrm{~m}}} \vec{u}_{\mathrm{visc}}=-\frac{x_{j} c_{\mathrm{t}} R T B_{0}}{\eta D_{j \mathrm{~m}}} \vec{\nabla} P$

and finally arrived at their equation

$\sum_{j=1}^{n} \frac{x_{i} x_{j}}{D_{i j}}\left(\vec{u}_{i}-\vec{u}_{j}\right)+\frac{x_{i}}{D_{i \mathrm{~m}}} \vec{u}_{i}=-\frac{x_{i}}{R T}\left(\vec{\nabla}_{T} \mu_{i}\right)-\frac{x_{i} B_{\mathrm{o}}}{\eta D_{i \mathrm{~m}}} \vec{\nabla} P$

There is an inconsistency in this derivation. The assumed condition for the pure component is

$\vec{\nabla}_{T} \mu_{\mathrm{s}}=\overrightarrow{0}$

However, for a single component

$\vec{\nabla}_{T} \mu_{\mathrm{s}}=\vec{V}_{\mathrm{s}} \vec{\nabla} P$

holds, and so Eq. (64) implies

$\vec{\nabla} P=\overrightarrow{0}$

and

$\vec{u}_{\text {visc }}=\overrightarrow{0}$

This is the consequence of trying to talk an extra viscous term into an equation which already contains the same term. Again it is concluded that the resulting equation for liquids (Eq. (63)) is incorrect.

The question then remains how to proceed from Bearman's equation (Eq. (54)) or the transformed form (Eq. (56)). If we only apply Eq. (56) for the fluid mixture, we will have the membrane as an external body. Eq. (59) is then just the Navier-Stokes equation for the mixture. When integrating over the pore cross-section, assuming negligible acceleration and inertial effects, in the integrated $\sum_{j=1}^{n} \vec{B}_{j}$ term we will have the viscous force of the membrane walls on the mixture. The mechanical equilibrium of the fluid then requires that the sum of the pressure, external and wall friction forces is zero. Thus it seems reasonable to consider $\vec{B}_{j}$ as the effect of the viscous drag of the wall on component $j$. In the flow region considered, it seems plausible to take this drag linear in the component velocity, and therefore we have

$\vec{B}_{j}=g_{j \mathrm{~m}} \vec{u}_{j}$

in which a friction factor between the component and the membrane appears.

By means of the substitution

$g_{i \mathrm{~m}}=c_{\mathrm{t}} R T \frac{x_{i}}{D_{\mathrm{im}}}$

the integrated Eq. (33) then becomes

$\sum_{j=1}^{n} \frac{x_{i} x_{j}}{D_{i j}}\left(\vec{u}_{i}-\vec{u}_{j}\right)+\frac{x_{i}}{D_{i \mathrm{~m}}} \vec{u}_{i}=-\frac{x_{i}}{R T}\left(\vec{\nabla}_{r} \mu_{i}\right)$

which is identical to the Lightfoot equation. Thus the results of the statistical mechanical treatment are identical to those derived from irreversible thermodynamics. 
In the foregoing analysis, the body forces have been left out; in the original derivation these were present. However, following the steps of Mason and Viehland [3], with the terms included, gives the same contradiction as illustrated by Eq. (67).

It is concluded that the DGM equations which include the extra viscous flux term are incorrect due to derivational errors and provide double counting of the viscous terms. In gas transport in the transition region, the success of the DGM equations is due to a compensation of errors: on the one hand, an extra viscous flux term is added, and on the other, the $D^{\prime}{ }_{\text {im }}$ term, which is clearly a viscous friction term, is arbitrarily deprived of its viscous nature and is turned into a Knudsen coefficient [30]. Although we could live with the final result for transition situations involving single gases, for gas mixtures, especially at low Knudsen numbers, incorrect results will be obtained.

Another consequence of the foregoing analysis is that the diffusion coefficients in the Lightfoot equation are not "augmented" but real diffusion coefficients, and the lengthy discussions, e.g. in Refs. [2,9], are essentially due to the errors in the DGM equations.

With regard to terminology, the concept of "parallel diffusive flow and viscous flow" in porous systems is erroneous or meaningless, since it is a distortion of the expression of the mass flux with respect to the mass-averaged velocity (Eq. (47)). For porous systems, it clearly follows from the solutions for the gas and liquid models that, in the transport equations, both viscous friction and intermolecular diffusion terms appear and they cannot be separated.

\section{Intramembrane transport: the binary friction model for liquids}

In this section, we consider the permeation of liquids for which the mean free path is much smaller than the pore diameter. We limit ourselves to the one-dimensional case, and so drop the spatial vector notation to avoid confusion with component-space vectors and tensors. Furthermore, we consider cylindrical pores; in Section 5 we include porosity and tortuosity corrections for transport over the whole membrane area. In addition, we assume the absence of external forces, and neglect gravity. We start from the Lightfoot equation (Eq. (13)) and for the fluid keep the MaxwellStefan diffusion coefficients. For the friction between the components and the membrane, we assume that it may be split up into a membrane geometry-dependent part $\alpha_{m}$ and a component-dependent part, and that, in the latter, we have a proportionality to the volume fraction of the components. Thus we transform

$r_{i \min } N_{i}=\alpha_{\mathrm{m}} \kappa_{i} \phi_{i} u_{i}$

and obtain the expression

$\frac{x_{i}}{R T} \nabla_{T, P} \mu_{i}+\frac{c_{i} \bar{V}_{i}}{c_{\mathrm{t}} R T} \nabla P=\sum_{j=1}^{n} \frac{x_{i} N_{j}-x_{j} N_{i}}{c_{\mathrm{t}} \boxplus_{i j}}-\alpha_{\mathrm{m}} \kappa_{i} \phi_{i} u_{i}$
Summing over all components, we obtain

$\frac{1}{c_{\mathrm{t}} R T} \nabla P=-\alpha_{\mathrm{m}} \sum_{i=1}^{n} \kappa_{i} \phi_{i} u_{i}$

Now let us regard the hypothetical concept of a homogeneously flowing mixture, in which all components move with the same pore-averaged velocity. From a macroscopic engineering point of view, this is the situation we always assume for the laminar transport of fluids in capillaries or the motion of mixtures in a rheometer, and for all intents and purposes we regard this as viscous flow. We can integrate the NavierStokes equations with the appropriate boundary conditions to solve the flow pattern and integrate that to one average velocity for the mixture. Let us for a moment assume that in systems with very small pores, but outside the Knudsen regime, by some kind of demon or mechanism there will also be the possibility of creating this uniform molecular velocity pattern. Then we will also have a purely viscous flow. We call this hypothetical situation "homogeneous viscous mixture flow (HVMF)".

For such a flow, we can use the Darcy equation

$\nabla P_{\mathrm{HVMF}}=-\frac{\eta^{\mathrm{p}}}{B_{\mathrm{o}}} u^{\mathrm{v}}=-\frac{\eta^{\mathrm{p}}}{B_{\mathrm{o}}} \frac{N_{\mathrm{t}}}{c_{\mathrm{t}}}$

in which the viscosity $\eta^{p}$ is the viscosity of the mixture as it manifests itself in the pores; for pores larger than a few molecular diameters, this viscosity approaches the value measured in a rheometer.

From Eq. (73), we have

$$
\eta^{\mathrm{p}}=\alpha_{\mathrm{m}} B_{\mathrm{o}} c_{\mathrm{t}} R T \sum_{i=1}^{n} \kappa_{i} \phi_{i}
$$

Since we have taken $\alpha_{\mathrm{m}}$ to be dependent only on the membrane structure, it follows that

$\alpha_{\mathrm{m}}=1 / B_{\mathrm{o}}$

and

$\eta^{\mathrm{p}}=c_{\mathrm{t}} R T \sum_{i=1}^{n} \kappa_{i} \phi_{i}$

Since the HVMF assumption is used in the interpretation of viscosity measurements, $\kappa_{i}$ follow from the measured relation between the composition and mixture viscosity; we denote $\kappa_{i}$ as the "fractional viscosity coefficients".

For a mixture with different species velocities, we assume that, at a given composition, the friction between the component and the wall is accounted for in the last term of Eq. (72), but that the value of $\kappa_{i}$ is equal to that in the HVMF situation for the prevailing $T$ and $P$. The physical idea is that the friction between different species is accounted for in the Maxwell-Stefan diffusion term, and the friction between a species and the wall is only influenced by the species type, its velocity with respect to the wall and its concentration, irrespective of the other species velocities. This is no different from the assumptions generally made for the interspecies 


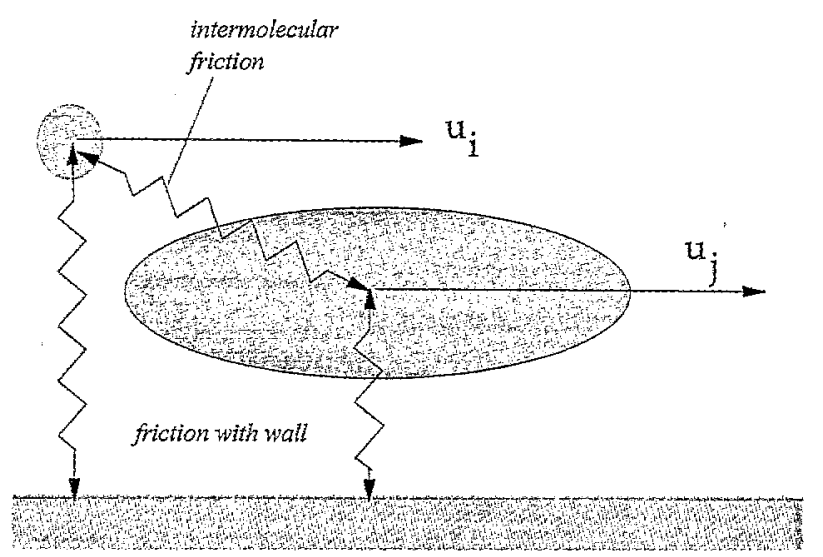

Fig. 1. Schematic representation of forces acting between molecules and between molecules and a solid wall.

friction coefficients, as represented in $\bigoplus_{i j}$, which are assumed to depend on the composition, pressure and temperature, but not on the fluxes. A schematic view of the forces is given in Fig. 1.

Substituting Eq. (76) into Eq. (72) gives

$\frac{x_{i}}{R T} \nabla_{T, P} \mu_{i}+\frac{c_{i} \bar{V}_{i}}{c_{\mathrm{t}} R T} \nabla P=\sum_{j=1}^{n} \frac{x_{i} N_{j}-x_{j} N_{i}}{c_{\mathrm{t}} D_{i j}}-\frac{1}{B_{\mathrm{o}}} \kappa_{i} \phi_{i} u_{i}$

The usual next step in the derivation is the development into molar fluxes and gradients of the mole fraction, and this is performed below. In our experience, the numerical solution of steady state equations is rather cumbersome, and here we choose a finite difference method, which solves the transient transport equations, in order to obtain the final steady state. The numerical solution technique employed requires that the continuity equation is expressed in terms of the concentrations rather than the mole fractions, and we derive these equations as well. This has the obvious advantage that the analysis of transient phenomena is included.

For the gradient of the chemical potential, we can write [23,36]

$c_{\mathrm{t}} \frac{x_{i}}{R T} \nabla_{T, P} \mu_{i}=c_{\mathrm{t}} \sum_{j=1}^{n-1} \Gamma_{i j} \nabla x_{j}$

and we develop the diffusion term in Eq. (78) towards $(n-1)$ fluxes. Since we have already chosen the membrane as the frame of reference, and we have an expression for the total flux, we eliminate the flux of component $n$ by

$N_{n}=N_{\mathrm{t}}-\sum_{j=1}^{n-1} N_{j}$

From Eq. (78) and the expression of the right-hand side in terms of the $(n-1)$ component fluxes and the total flux, it now follows, as derived in Appendix B, that

$c_{\mathrm{t}}[\mathbf{\Gamma}](\nabla x)-N_{\mathrm{t}}[\mathbf{F}](x)=-[\mathbf{G}](N)$

in which

$$
\begin{aligned}
& {[\boldsymbol{\Gamma}]_{i j}=\Gamma_{i j}=\delta_{i j}+x_{i} \frac{\partial \ln \gamma_{i}}{\partial x_{j}}} \\
& {[\mathbf{F}]_{i j}=\delta_{i j}\left\{\frac{c_{\mathrm{t}}^{2} \bar{V}_{i} \bar{V}_{n}}{B_{0}} \kappa_{n}+\frac{1}{\theta_{i n}}\right\}} \\
& {[\mathbf{G}]_{i j}=[\mathbf{B}]_{i j}+\delta_{i j} \frac{\kappa_{i} c_{\mathrm{t}} \bar{V}_{i}}{B_{0}}-\frac{c_{\mathrm{t}}^{2}}{B_{0}} x_{i} \bar{V}_{i}\left(\kappa_{j} \bar{V}_{j}-\kappa_{n} \bar{V}_{n}\right)} \\
& {[\mathbf{B}]_{i i}=\frac{x_{i}}{D_{i n}}+\sum_{k=1 k \neq i}^{n} \frac{x_{k}}{D_{i k}}} \\
& {[\mathbf{B}]_{i j}=-x_{i}\left(\frac{1}{D_{i j}}-\frac{1}{D_{i n}}\right) i \neq j}
\end{aligned}
$$

and so [B] is identical to the Maxwell-Stefan friction matrix in the classical development.

Inversion of the generalized friction matrix [G] then gives the expression for the fluxes

$(N)=-c_{t}[\mathbf{G}]^{-1}[\Gamma](\nabla x)+N_{t}[\mathbf{G}]^{-1}[\mathbf{F}](x)$

For large pores, in which $B_{0}$ tends to infinity, we obtain

$$
\begin{aligned}
& {[\mathbf{G}]_{i j}^{\infty}=[\mathbf{B}]_{i j}} \\
& {[\mathbf{F}]_{i j}^{\infty}=\delta_{i j} \frac{1}{\bigoplus_{i n}}}
\end{aligned}
$$

and thus

$$
(N)^{\infty}=-c_{\mathrm{t}}[\mathbf{B}]^{-1}[\Gamma](\nabla x)+N_{\mathrm{t}}[\mathbf{B}]^{-1}[\mathbf{F}]^{\infty}(x)
$$

As derived in Appendix $\mathrm{C}$

$$
[\mathbf{F}]^{\infty}(x)=[\mathbf{B}](x)
$$

from which follows

$$
(N)^{\infty}=-c_{\mathrm{t}}[\mathbf{B}]^{-1}[\Gamma](\nabla x)+N_{\mathrm{t}}(x)
$$

the result of Taylor and Krishna [23].

\subsection{Results for a binary mixture}

For $n=2$, we develop from Eq. (81)

$$
\begin{aligned}
& c_{\mathrm{t}} \Gamma_{1} \nabla x_{1}-N_{\mathrm{t}} x_{1}\left(\frac{c_{\mathrm{t}}^{2} \bar{V}_{1} \bar{V}_{2}}{B_{\mathrm{o}}} \kappa_{2}+\frac{1}{D_{12}}\right) \\
& =-N_{1}\left(\frac{x_{1}}{D_{12}}+\frac{x_{2}}{D_{12}}+\frac{\kappa_{1} c_{\mathrm{t}} \bar{V}_{1}}{B_{\circ}}-\frac{c_{\mathrm{t}}^{2} x_{1} \bar{V}_{1}}{B_{\circ}}\left(\kappa_{1} \bar{V}_{1}-\kappa_{2} \bar{V}_{2}\right)\right)
\end{aligned}
$$

Rearrangement gives

$$
N_{1}=\frac{-c_{\mathrm{t}} \Gamma_{1} \nabla x_{1}+N_{\mathrm{t}} x_{1}\left(\frac{1}{D_{12}}+\frac{c_{\mathrm{t}}^{2} \bar{V}_{1} \bar{V}_{2}}{B_{0}} \kappa_{2}\right)}{\frac{1}{D_{12}}+\frac{c_{\mathrm{t}}}{B_{\mathrm{o}}}\left(\phi_{2} \kappa_{1} \bar{V}_{1}+\phi_{1} \kappa_{2} \bar{V}_{2}\right)}
$$

and the pressure gradient is given by

$$
\nabla P=-\frac{c_{\mathrm{t}} R T}{B_{0}}\left(\kappa_{1} N_{1} \bar{V}_{1}+\kappa_{2} N_{2} \bar{V}_{2}\right)
$$


For large pores and large $B_{0}$, we easily obtain

$N_{1}=-c_{\mathrm{t}} \nexists_{12} \Gamma_{1} \nabla x_{1}+N_{\mathrm{t}} x_{1}$

corresponding to the formulation of Bird et al. [34].

For the case of a virtually pure liquid, $x_{1} \rightarrow 1$, Eq. (94) changes to Darcy's law and Eq. (93) changes to an identity.

\subsection{Driving force on concentration basis}

We convert the gradient of the chemical potential as follows

$c_{\mathrm{i}} \frac{x_{i}}{R T} \nabla_{T, P} \mu_{i}=\sum_{j=1}^{n-1} \Gamma_{c_{,}, j} \nabla c_{j}$

in which $\Gamma_{\mathrm{c}}$ is given by

$\left[\Gamma_{\mathrm{c}}\right]_{i j}=\Gamma_{\mathrm{c}, \mathrm{ij}}=\delta_{i j}+c_{i} \frac{\partial \ln \gamma_{i}}{\partial c_{j}}-\frac{c_{i}}{c_{\mathrm{t}}}\left(1-\frac{\bar{V}_{j}}{\bar{V}_{n}}\right)$

In the diffusion term, we substitute expressions for $c$ in terms of the mole fraction, and after some derivation obtain

$(N)=-[\mathbf{G}]^{-1}\left[\boldsymbol{\Gamma}_{\mathrm{c}}\right](\nabla c)+u^{v}[\mathbf{G}]^{-1}[\mathbf{F}](c)$

in which $[\mathbf{G}]$ and $[\mathbf{F}]$ are identical to those defined in Eqs. (82)-(85).

For the binary system, this changes to

$$
N_{1}=\frac{-\Gamma_{c, 1} \nabla c_{1}+u^{\mathrm{v}} c_{1}\left(\frac{1}{D_{12}}+\frac{c_{\mathrm{l}}^{2} \bar{V}_{1} \bar{V}_{2}}{B_{\mathrm{o}}} \kappa_{2}\right)}{\frac{1}{D_{12}}+\frac{c_{\mathrm{t}}}{B_{\mathrm{o}}}\left(\phi_{2} \kappa_{1} \bar{V}_{1}+\phi_{1} \kappa_{2} \bar{V}_{2}\right)}
$$

\subsection{Estimation of the fractional viscosity coefficients for solutions}

If we have a sufficiently large pore, we would expect that

$\eta^{\mathrm{p}}=c_{\mathrm{r}} R T \sum_{i=1}^{n} \kappa_{i} \phi_{i} \approx \eta$

and so from the measured free solution viscosity data as a function of concentration, a first estimate of $\kappa_{i}$ can be made. In general, this can be done by means of regression of the viscosity vs. composition, from which the concentrationdependent $\kappa_{i}$ follow.

For a binary system, we have

$$
\eta \approx c_{\mathrm{t}} R T\left(\kappa_{1} \phi_{1}+\kappa_{2} \phi_{2}\right)=c_{\mathrm{t}} R T\left\{\phi_{1}\left(\kappa_{1}-\kappa_{2}\right)+\kappa_{2}\right\}
$$

An approximation for dilute solutions may involve the following method. We take component 2 as the solvent, and we further assume that we may relate $\kappa_{2}$ directly to the pure solvent (water) viscosity

$\eta_{\text {soivent }}=\eta_{2}=c_{t} R T_{\kappa_{2}}$

From this we find for $\kappa_{1}$ as an approximation

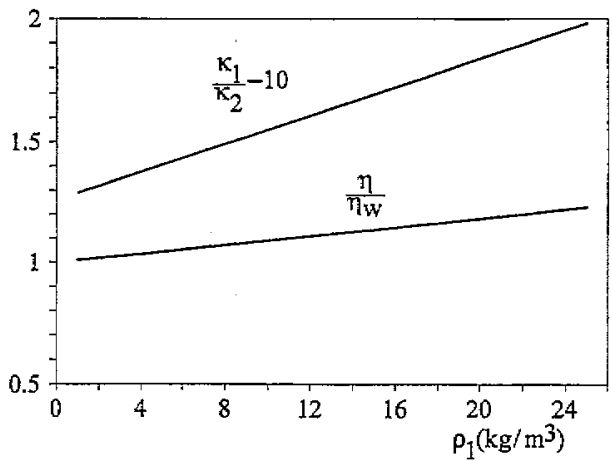

Fig. 2. Viscosity $\eta$ and fractional viscosity coefficient $\kappa$ of PEG-3400 solutions as a function of the PEG concentration.

$\frac{\kappa_{1}}{\kappa_{2}}=1+\frac{1}{\phi_{1}}\left(\frac{\eta-\eta_{2}}{\eta_{2}}\right)=1+\frac{\eta_{\mathrm{sp}}}{\phi_{1}}$

and so $\kappa_{1}$ can be calculated from the measured viscosity data.

In Fig. 2, the values of $\kappa_{1} / \kappa_{2}$ are presented, as calculated from experimental viscosity data for aqueous solutions of PEG-3400, together with the viscosity data (Thomas data as given by van Oers [15]). Over the concentration range, there is a slight increase in viscosity and also a slight increase in $\kappa_{1} / \kappa_{2}$, because the mole fraction of PEG is only very low. However, the absolute value of about 11-12 indicates that the PEG molecules experience a considerable friction near a (pore) wall.

\section{Mass transfer in the boundary layer applied to a tubular membrane}

Here the development is followed as given by Krishna [32] and Taylor and Krishna [23]. We write the general equation

$(N)=-c_{\mathrm{t}}\left\{[\mathbf{D}]+[\mathbf{D}]_{\text {turb }}\right\} \nabla(x)+N_{\mathrm{t}}(x)$

with again the membrane as fixed reference frame.

In Eq. (10)4, [D] is the Fickian molecular diffusion matrix, which is equal to

$[\mathbf{D}]=[\mathbf{B}]^{-1}[\Gamma]$

The turbulent diffusion matrix is given by

$[\mathbf{D}]_{\text {turb }}=D_{\text {turb }}[\mathbf{I}]$

The basic assumption is that we may use this approach also in the case of permeation.

For the turbulent diffusivity, we also assume the estimate

$\mathrm{Sc}_{\text {turb }}=\frac{\nu_{\text {turb }}}{D_{\text {turb }}} \approx 1$

By definition, the turbulent kinematic viscosity is given by

$\frac{\nu_{\text {urb }}}{\nu} \approx \frac{1-\left(y^{+} / R_{\mathrm{w}}^{+}\right)}{\mathrm{d} u^{+} / \mathrm{d} y^{+}}-1$

which for most situations changes to 
$\frac{\nu_{\text {turb }}}{\nu} \approx \frac{1}{\mathrm{~d} u^{+} / \mathrm{d} y^{+}}-1$

The dimensionless variables are defined as

$y^{+}=y u^{*} / \nu$

$R_{\mathrm{w}}^{+}=\operatorname{Re} \sqrt{f / 8}$

$u^{*}=\left\langle u_{\mathrm{t}}\right\rangle \sqrt{f / 2}$

$u^{+}=u / u^{*}$

in which $f$ is the Fanning friction factor defined by

$\Delta p=4 f \frac{1}{2} \rho u_{\mathrm{r}}^{2}\left(\frac{L}{d}\right)_{\text {tube }}$

Let us first consider the zero flux case for constant diffusion coefficient. The zero flux mass transfer coefficient can be written as

$\frac{1}{k}=\int_{0}^{y \mathrm{~b}} \frac{\mathrm{d} y}{D_{\mathrm{m}}+D_{\text {turb }}}$

in which $y=(R-r)$ is the distance from the tube wall and $y_{\mathrm{b}}$ is the distance to the bulk over which concentration gradients appear. In dimensionless form, this changes to

$\mathrm{St}^{-1}=\sqrt{\frac{2}{f}} \int_{0}^{y+} \frac{\mathrm{d} y^{+}}{(1 / \mathrm{Sc})+\left(\nu_{\text {turb }} / \nu\right)}$

where $\mathrm{St}$ is the Stanton number and equals $k /\left\langle u_{\mathrm{t}}\right\rangle$, and $\left\langle u_{\mathrm{t}}\right\rangle$ is the averaged velocity along the wall.

From the definitions of the dimensionless quantities, we also have

$u^{+}=\int_{0}^{y_{0}^{+}} \frac{\mathrm{d} y^{+}}{1+\left(\nu_{\text {turb }} / \nu\right)}$

Several models have been proposed in the literature for the relation between the turbulent viscosity and the dimensionless velocity and the distance from the wall. Taylor and Krishna [23] worked out the von Karman model in which, in the laminar sublayer, it is assumed that

$\nu_{\mathrm{t}}=0 \quad 0<y^{+}<5$

which works well at Sc numbers near unity. They also showed a considerable deviation for high Sc numbers between the results of the von Karman model and the Chilton-Colburn analogy. They referred to the work of Vieth, who showed that the Chilton-Colburn analogy corresponds to a turbulent viscosity relation

$\frac{\nu_{\text {turb }}}{\nu}=c_{\text {Vieth }}\left(\frac{f}{2}\right)^{3 / 2}\left(y^{+}\right)^{3}$

The constant is equal to

$c_{\text {Vieth }}=\left(\frac{2}{9} \pi \sqrt{3}\right)^{3}=1.768$
(It should be noted that the exponent of $f$ should be $3 / 2$ instead of the value of $2 / 3$ mentioned in Ref. [23].)

Since in ultrafiltration, we typically have Sc numbers of the order of $10^{4}$, strong deviations between the two theories may be expected, without any difference in the velocity profiles; this follows directly from inspection of the integrands of Eq. (113) and Eq. (114). Another model for the turbulent viscosity is that of Deissler (Bird et al. [34])

$\frac{\mathrm{d} u^{+}}{\mathrm{d} y^{+}}=\frac{1}{1+n^{2} u^{+} y^{+}\left[1-\exp \left(-n^{2} u^{+} y^{+}\right)\right]}$

where $n=0.124$ is the Deissler constant. Integration of Eq. (112) leads to a flow distribution which also fits very well with the experimental data close to the wall.

From Eq. (117), for the turbulent viscosity

$\frac{\nu_{\text {turb }}}{\nu}=n^{2} u^{+} y^{+}\left[1-\exp \left(-n^{2} u^{+} y^{+}\right)\right]$

Taylor and Krishna [23] also treat the mixing length approximation of van Driest

$\frac{\nu_{\text {turb }}}{\nu}=\left(l^{+}\right)^{2}\left|\frac{\mathrm{d} u^{+}}{\mathrm{d} y^{+}}\right|$

with

$l^{+}=\lambda y^{+}\left[1-\exp \left(-y^{+} / A^{+}\right)\right]$

in which $\lambda=0.4$ and $A^{+}=26$, and from theory it follows that

$\left|\frac{\mathrm{d} u^{+}}{\mathrm{d} y^{+}}\right|=\frac{-1+\sqrt{1+4\left(l^{+}\right)^{2}}}{2\left(l^{+}\right)^{2}}$

and so

$\frac{\nu_{\text {turb }}}{\nu}=\frac{1}{2}\left(\sqrt{1+4\left(l^{+}\right)^{2}}-1\right)$

Before applying this to the membrane polarization layer, a comparison of the predicted Sh numbers was made as a function of the Re and Sc numbers for the zero flux condition and constant properties. For the friction factor, the Blasius equation for smooth walls was taken

$4 f=0.3164 \mathrm{Re}^{-0.25}$

Numerical integration over distances up to $y^{+}=50$ showed that, in all cases, integration up to $y^{+}=5$ was sufficient to obtain convergence in the integral, except for the von Karman model. Typical results for $\mathrm{Re}=10^{4}$ are given in Fig. 3; calculations for other $\operatorname{Re}$ numbers showed similar results. As expected, the Vieth model covers the Chilton-Colburn analogy; the van Driest and Deissler models give virtually identical results, which are slightly lower than the ChiltonColburn values. The von Karman model predicts no dependence of Sh on Sc; this is caused by Eq. (115), which leads to

$$
\begin{aligned}
& \mathrm{St} \approx a \cdot \mathrm{Sc}^{-1} \\
& \mathrm{Sh}=\mathrm{St} \cdot \mathrm{Re} \cdot \mathrm{Sc} \neq f(\mathrm{Sc})
\end{aligned}
$$




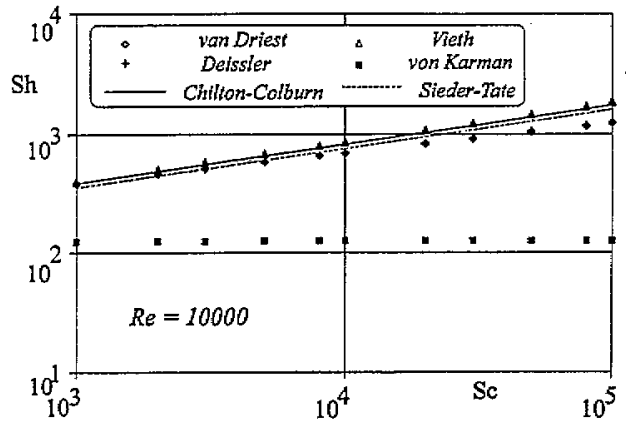

Fig. 3. Comparison of Sh number obtained from yarious turbulence models and two correlations, as a function of the $\mathrm{Sc}$ number, for $\mathrm{Re}=10^{4}$.

Also shown is the correlation of Sieder and Tate [37]

$\mathrm{Sh}=0.023 \mathrm{Re}^{0.8} \mathrm{Sc}^{0.33}$

The results are seen to be somewhat lower than those of the Chilton-Colburn analogy.

However, application in the work of van Oers [15] in tubular module ultrafiltration showed that a value of 0.027 for the pre-factor gives a better coverage with the experimental results, and application of this pre-factor increases the agreement with the Chilton-Colburn analogy.

Since experience has shown that the Chilton-Colburn analogy is very successful in predicting heat and mass transfer rates, we decided to take the Vieth approximation as the basis for the boundary layer theory in the case of variable properties, and the region $y^{+} \leq 5$ as the polarization layer.

\section{Combined model for ultrafiltration including both the membrane and polarization layer, and comparison with experiment}

For one-dimensional transport in the polarization layer, we write a Fickian-type equation for the flux per unit area of the membrane in concentration terms

$(N)=-\left\{[\mathbf{D}]+[\mathbf{D}]_{\text {turb }}\right\} \frac{\partial}{\partial z}(c)+u^{v}(c)$

and the Fickian molecular diffusion matrix is given by

$[\mathrm{D}]=[\mathbf{B}]^{-1}\left[\boldsymbol{\Gamma}_{\mathrm{c}}\right]$

The transport inside the membrane is now described with fluxes per unit membrane area and concentrations $c^{\prime}$ in the liquid phase in the pores. In view of the porosity and tortuosity, we have

$(N)=\frac{\epsilon}{\tau}\left(N^{\prime}\right)$

in which $N^{\prime}$ are the fluxes inside the membrane per unit of pore area. Therefore we have

$(N)=-\left[\mathbf{G}^{\prime}\right]^{-1}\left[\boldsymbol{\Gamma}_{\mathfrak{c}}\right] \frac{\partial}{\partial z}\left(c^{\prime}\right)+u^{v}\left[\mathbf{G}^{\prime}\right]^{-1}\left[\mathbf{F}^{\prime}\right]\left(c^{\prime}\right)$ with

$$
\begin{aligned}
& {\left[\mathbf{G}^{\prime}\right]=\frac{\tau}{\epsilon}[\mathbf{G}]} \\
& {\left[\mathbf{F}^{\prime}\right]=\frac{\tau}{\epsilon}[\mathbf{F}]}
\end{aligned}
$$

We also write Eq. (129) in Fickian form

$$
(N)=-\left[\mathbf{S}^{\prime}\right] \frac{\partial}{\partial z}\left(c^{\prime}\right)+u^{\mathrm{v}}\left[\mathbf{K}^{\prime}{ }_{\mathrm{v}}\right]\left(c^{\prime}\right)
$$

with

$$
\begin{aligned}
& {\left[\mathbf{S}^{\prime}\right]=\left[\mathbf{G}^{\prime}\right]^{-1}\left[\boldsymbol{\Gamma}_{c}\right]} \\
& {\left[\mathbf{K}_{\mathrm{v}}^{\prime}\right]=\left[\mathbf{G}^{\prime}\right]^{-1}\left[\mathbf{F}^{\prime}\right]}
\end{aligned}
$$

We have the continuity equations

$\frac{\partial}{\partial t}(c)=-\frac{\partial}{\partial z}(N)$ for the polarization layer

$\epsilon \frac{\partial}{\partial t}\left(c^{\prime}\right)=-\frac{1}{\tau} \frac{\partial}{\partial z}(N)$ inside the membrane

As boundary conditions, we have

$$
\begin{aligned}
& z=-\delta_{\mathrm{pol}},(c)=\left(c_{\mathrm{b}}\right) \\
& z=L_{\mathrm{m}},(N)=u^{v}\left(c_{\mathrm{p}}\right),\left(c^{\prime}\right)=\left[\mathbf{K}_{\mathrm{eq}}\right]\left(c_{\mathrm{p}}\right)
\end{aligned}
$$

in which $\left(c_{\mathrm{b}}\right)$ and $\left(c_{\mathrm{p}}\right)$ are the bulk and permeate concentrations and $\left[K_{e q}\right]$ is the equilibrium matrix

$$
\begin{aligned}
& {\left[\mathrm{K}_{\mathrm{eq}}\right]_{i j}=\delta_{i j} K_{\mathrm{eq}, i}} \\
& K_{\mathrm{eq}, i} \equiv c^{\prime}{ }_{i} / c_{i}
\end{aligned}
$$

At the interface, $z=0$, we assume equilibrium.

The driving force for flow is given by

$\Delta P_{\text {flow }}=\Delta P_{\text {tot }}-\sigma \Delta \Pi$

in which $\Delta \Pi$ is the osmotic pressure difference across the membrane, dependent on the concentrations on both sides, and $\sigma$ is the osmotic reflection coefficient.

The relationship between the superficial velocity and effective pressure drop follows as

$\Delta P_{\text {flow }}=\frac{\tau R T}{\epsilon B_{\mathrm{o}}} \int_{0}^{L_{\mathrm{m}}} c_{\mathrm{t}} \sum_{i=1}^{2} \kappa_{i} N_{i} \bar{V}_{i} \mathrm{~d} z$

It follows that the membrane resistance determined from the pure water flux

$R_{\mathrm{m}}=\frac{\Delta p}{\eta_{\mathrm{w}} u_{\mathrm{w}}^{\mathrm{v}}}$

equals

$R_{\mathrm{m}}=\frac{L_{\mathrm{m}}}{B_{\mathrm{o}}} \frac{\tau}{\epsilon}$

from which the ratio $\epsilon / \tau$ can be calculated. 


\subsection{Membrane filtration of PEG-3400 solutions}

Van Oers [15] and Meeuwsen [38] performed experiments on the transport of PEG-3400 in ultrafiltration in a 1.8 $\mathrm{m}$ tubular module. They varied the transmembrane pressure and measured the flux through the membrane and the apparent retention of PEG for circulation velocities through the module of 1.04 and $1.95 \mathrm{~m} \mathrm{~s}^{-1}$. They divided the permeate side into four different compartments and established that, in the last two compartments, identical results were obtained; no entrance effects played a role. Van Oers modelled the results with the dusty gas-type Maxwell-Stefan equation, and took the model of a stagnant film for the polarization layer. She solved the steady state equations numerically and applied non-linear regression to obtain a good fit with the experimental data. Five parameters had to be fitted: the pre-factor in the Sieder-Tate relationship, $\epsilon / \tau, K_{\mathrm{eq}}, D_{\mathrm{wm}}$ and $D_{\mathrm{sm}}$.

Here we write the transport equations in binary form

$\frac{\partial c_{1}}{\partial t}=-\frac{\partial}{\partial z}\left(N_{1}\right)$

with

$N_{1}=-\left(D+D_{\mathrm{t}}\right) \frac{\partial c_{1}}{\partial z}+u^{\mathrm{v}} c_{1}$

$D=D_{12} \Gamma_{\mathrm{cl}}$

For the membrane, we have

$\epsilon \frac{\partial c_{1}{ }^{\prime}}{\partial t}=-\frac{1}{\tau} \frac{\partial}{\partial z}\left(N_{1}^{\mathrm{m}}\right)$

with

$N_{1}^{\mathrm{m}}=-\frac{\Gamma_{\mathrm{c} 1}}{G_{\mathrm{m} 1}^{\prime}} \frac{\partial c^{\prime}}{\partial z}+u_{1}^{\mathrm{v}} \frac{F_{\mathrm{m}}^{\prime}}{G_{\mathrm{m} 1}^{\prime}} c^{\prime}{ }_{1}$

where $\mathrm{N}_{1}^{\mathrm{m}}$ is the flux per square metre of membrane area

$$
\begin{aligned}
& G_{\mathrm{ml}}^{\prime}=\left(\frac{1}{D_{12}}+\frac{c_{\mathrm{t}}^{2} \bar{V}_{1} \bar{V}_{2}}{B_{\mathrm{o}}} \kappa_{2}\right) \frac{\tau}{\epsilon} \\
& F_{\mathrm{m}}^{\prime}=\left(\frac{1}{D_{12}}+\frac{c_{\mathrm{t}}}{B_{\mathrm{o}}}\left(\phi_{2} \kappa_{1} \bar{V}_{1}+\phi_{1} \kappa_{2} \bar{V}_{2}\right) \frac{\tau}{\epsilon}\right.
\end{aligned}
$$

\subsection{Physical properties}

Most of the data here have been taken from the thesis of van Oers [15]:

The mole-averaged molecular mass of PEG-3400 is equal to 3600 and the true density is equal to $1204 \mathrm{~kg} \mathrm{~m}^{-3}$.

\subsubsection{Osmotic pressure}

The osmotic pressure was calculated according to

$$
\Pi=-\frac{R T}{\bar{V}_{\mathrm{w}}} \ln \left(a_{\mathrm{w}}\right)
$$

in which the water activity was calculated with the UNIQUAC model, with constants given by van Oers [15].

\subsubsection{Viscosity}

The following relation was used

$\eta_{\mathrm{sp}}=\frac{\eta-\eta_{\mathrm{w}}}{\eta_{\mathrm{w}}}=0.1(\eta)_{\mathrm{int}} \rho_{\mathrm{p}}+0.0033\left\{(\eta)_{\mathrm{int}} \rho_{\mathrm{p}}\right\}^{2}$

$(\eta)_{\text {int }}=6.04 \times 10^{-5} M_{n}^{0.90}$

in which $M_{\mathrm{n}}$ is the number-averaged molecular mass of PEG3400 (3158).

\subsubsection{Diffusion coefficient}

The molecular Fickian diffusion coefficient as measured by van der Linden (see [15]) is given as

$D=\left(5 \omega_{\mathrm{p}}+1.37\right) \times 10^{-10} \mathrm{~m}^{2} \mathrm{~s}^{-1}$

\subsubsection{Membrane resistance}

From the measured clean water flux, we obtain

$R_{\mathrm{m}}=3.558 \times 10^{12} \mathrm{~m}^{-1}$

\subsubsection{Membrane porosity and tortuosity}

No estimation of the porosity is needed. The dynamic change in the concentrations and fluxes depends on $\epsilon$, but the steady state values only depend on $\epsilon / \tau$, which is calculated from the membrane resistance and the supplier-provided pore diameter and membrane thickness according to Eq. (139). We obtain with $L_{\mathrm{m}}=5 \times 10^{-7} \mathrm{~m}$ and $d_{\text {pore }}=9 \times 10^{-9} \mathrm{~m}$, a value of $\epsilon / \tau=0.062$, which is about twice the value of van Oers.

\subsection{Numerical solution}

The system was solved by means of the numerical procedure PDESTEP for the non-steady state, starting with zero concentrations in the boundary layer and the membrane. The procedure uses a modified Crank-Nicholson scheme with variable time and distance steps. The flux was set as the independent parameter, and therefore the pressure drop follows from the integration of Eq. (137). After sufficient steps, steady state is reached, and the retention is calculated. In order to investigate the effects of osmotic pressure, the extreme values of the osmotic reflection coefficient $\sigma=0$ and $\sigma=1$ were used to calculate the total transmembrane pressure difference. The results for the retention are given in Fig. 4. Very good agreement is observed between the present model and the experimental data for a value of $K_{\mathrm{eq}}=0.69$. This is the only adjustable parameter needed! This is quite remarkable since no change was applied to $\Xi_{12}$ for the pore, and also free solution viscosity data were used to estimate the friction coefficients inside the membrane.

In Fig. 5, the relationship between the transmembrane pressure and the permeate flux is shown as determined from 


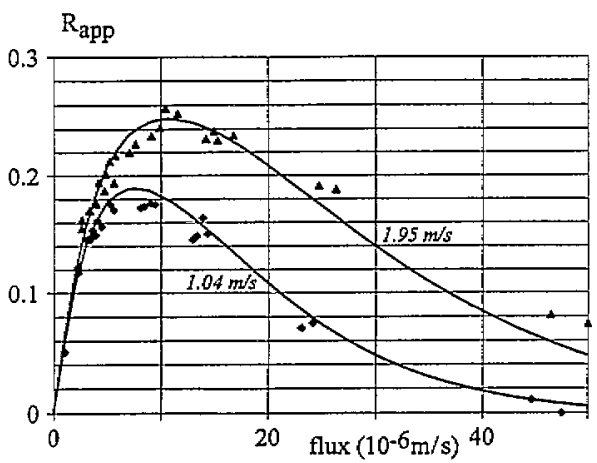

Fig. 4. Apparent retention of PEG-3400 in the ultrafiltration of aqueous solutions for $\rho=10 \mathrm{~kg} \mathrm{~m}^{-3}$ in a tubular module with a WFBX-0121 membrane for two different circulation velocities. Symbols, experimental data of van Oers [15] and Meeuwsen [38] for circulation velocities of 1.04 and $1.95 \mathrm{~m} \mathrm{~s}^{-1}$ respectively; drawn lines, from the present model.

experiments and from the present model. Also the clean water flux is shown. There is little difference between the results obtained for the extreme values of the osmotic reflection coefficients, and it seems that the values for $\sigma=1$ give a somewhat better coverage than those for $\sigma=0$. In view of the accuracy of measurement, however, it was felt that the only conclusions to be drawn at present are that there is good correspondence between the model and the experiments, and there is only a small effect from the osmotic pressure. The difference between the fluxes with PEG and with pure water can therefore be mainly attributed to the increased friction.

For completeness, in Fig. 6, the concentration profile in the boundary layer and the membrane is shown for a flux of $10^{-5} \mathrm{~m} \mathrm{~s}^{-1}$; it is clear that the assumed thickness of $y^{+}=5$ is sufficient to cover the region over which the concentration polarization takes place. In Fig. 7, the picture is expanded to show the concentration profile inside the membrane.

\section{The binary friction model for isothermal gases}

\subsection{Development of transport equations}

Contrary to liquid ultrafiltration, here we must take into account slip flow phenomena, such as Maxwell viscous slip and Kramers and Kistemaker's diffusive slip [31,33,35]. The model should describe the zero pressure limit of Knudsen flow in which no intermolecular collisions occur, the higher pressure limit for the normal region in which the Knudsen number $\mathrm{Kn}=\Lambda / r_{0}$ is very low and the intermediate slip flow regime.

We start again with the Lightfoot equation

$\frac{x_{i}}{R T} \vec{\nabla}_{T, P} \mu_{i}+\frac{\phi_{i}}{c_{\mathrm{t}} R T} \vec{\nabla} P=\sum_{j=1}^{n} \frac{\left(x_{i} \vec{N}_{j}-x_{j} \vec{N}_{i}\right)}{c_{\mathrm{t}} \Theta_{i j}}-r_{i \mathrm{~m}} \vec{N}_{i}$

We assume ideal gas behaviour and so

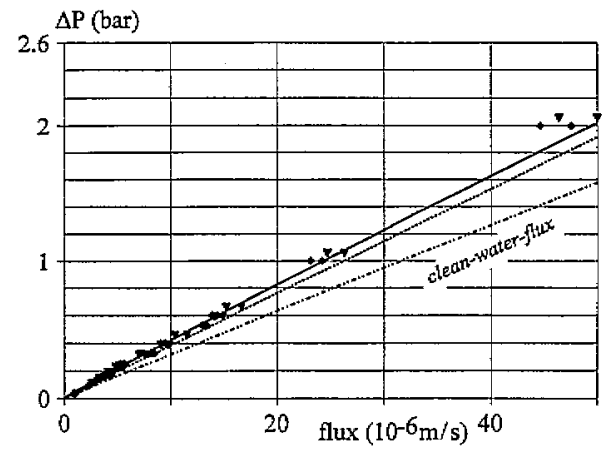

Fig. 5. Pressure drop vs, membrane flux for ultrafiltration. Same conditions as in Fig. 4. Drawn line according to present model for osmotic reflection coefficient of $\sigma=1$ and broken line for $\sigma=0$. Symbols, experimental data from van Oers [15] and Meeuwsen [38].

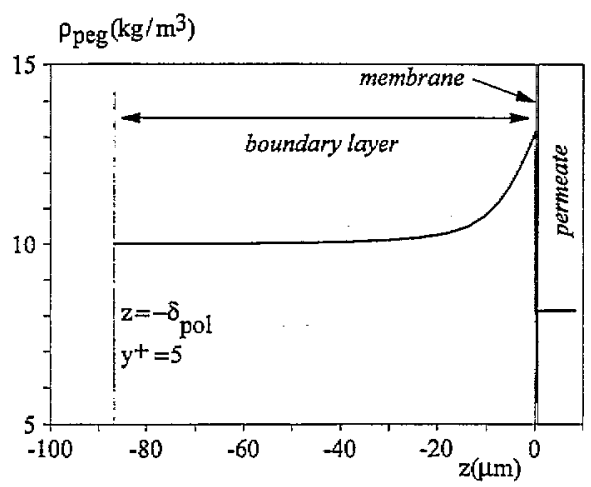

Fig. 6. Concentration profile for PEG-3400, calculated for a flux of $10^{-5} \mathrm{~m}$ $\mathrm{s}^{-1}$ at a circulation velocity of $1.04 \mathrm{~m} \mathrm{~s}^{-1}$.

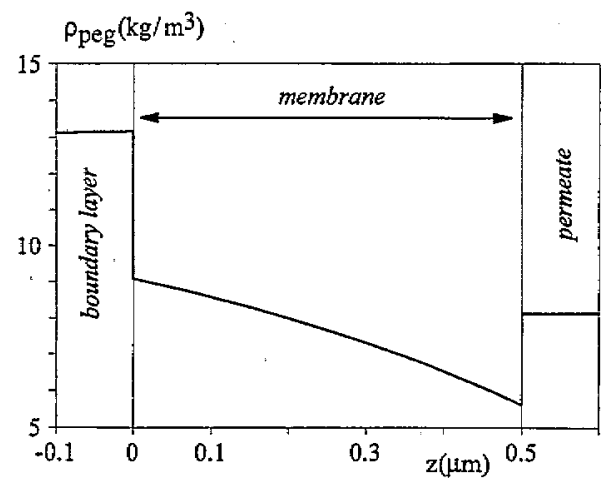

Fig. 7. Expanded view of Fig. 6 to show concentration profile inside the membrane.

$\frac{x_{i}}{R T} \vec{\nabla}_{T, P} \mu_{i}=\frac{x_{i}}{R T} R T \vec{\nabla}\left(\ln x_{i}\right)=\vec{\nabla} x_{i}$

$\phi_{i}=x_{i}$

$c_{\mathrm{t}}=\frac{P_{\mathrm{t}}}{R T}$

$c_{i}=x_{i} \frac{P_{\mathrm{t}}}{R T}$

We take the fluxes per unit area of pore along the pore path; Eq. (149) can then be written as 
$\vec{\nabla} x_{i}+\frac{x_{i}}{P_{\mathrm{t}}} \vec{\nabla} P_{\mathrm{t}}=R T \sum_{j=1}^{n} \frac{\left(x_{i} \vec{N}_{j}-x_{j} \vec{N}_{i}\right)}{P_{\mathrm{t}} \nexists_{i j}}-r_{i \mathrm{~m}} \vec{N}_{i}$

An alternative formulation is

$\frac{1}{P_{\mathrm{t}}} \vec{\nabla} P_{i}=R T \sum_{j=1}^{n} \frac{\left(x_{i} \vec{N}_{j}-x_{j} \vec{N}_{i}\right)}{P_{\mathrm{t}} D_{i j}}-r_{i \mathrm{~m}} \vec{N}_{i}$

Summing up over all components, the Maxwell-Stefan term cancels out, and we have

$\frac{1}{P_{\mathrm{t}}} \vec{\nabla} P_{\mathrm{t}}=-\sum_{j=1}^{n} r_{i \mathrm{~m}} \vec{N}_{i}$

\subsubsection{Single-component permeation}

Let us now first consider the case of a single pure gas. Maxwell [35] derived approximate relations for the so-called viscous slip assuming, among other things, the gas to be Maxwellian. His equation for flow in a capillary reads

$\vec{N}_{\mathrm{g}}=-\frac{r_{\mathrm{o}}^{2}}{8 \eta}\left(1+\frac{4 G}{r_{\mathrm{o}}}\right) \frac{P_{\mathrm{g}}}{R T} \frac{\mathrm{d} P_{\mathrm{g}}}{\mathrm{d} z}$

in which $G$ is the slip modulus ("Gleitmodulus"), which has the dimension of length. His theoretical estimate was

$G=\frac{2}{3}\left(\frac{2}{f}-1\right) \Lambda$

in which $f$ is the fraction of molecules diffusely reflected from the wall and $A$ is the mean free path. It is generally assumed that $f \approx 1$ [31]. From the values quoted by Maxwell of the experimental data of Kundt and Wartburg (see [35]), it can be deduced that $G / \Lambda=1.26$ for nitrogen and $G / \Lambda=1.31$ for hydrogen.

Knudsen experimentally studied the flow of gases through bundles of capillaries; his results for the flow of $\mathrm{CO}_{2}$ through capillaries of $0.02 \mathrm{~m}$ length and $33.3 \mu \mathrm{m}$ radius at $25^{\circ} \mathrm{C}$ are presented and discussed by Cunningham and Williams [31]. Knudsen's correlation may be written as $[8,31]$

$\vec{N}_{\mathrm{g}}=-\left(\frac{r_{\mathrm{o}}^{2}}{8 \eta}\left\langle P_{\mathrm{g}}\right\rangle+D_{\mathrm{g}}^{\mathrm{K}}\right) \frac{1}{R T} \frac{\Delta P_{\mathrm{g}}}{L}$

in which $\left\langle P_{\mathrm{g}}\right\rangle=\left\{P_{\mathrm{g}}(0)+P_{\mathrm{g}}(L)\right\} / 2$ is the average pressure, $L$ is the capillary length and $D_{\mathrm{g}}^{\mathrm{K}}$ is given by

$D_{\mathrm{g}}^{\mathrm{K}}=D_{\mathrm{go}}^{\mathrm{K}} \frac{1+c_{1}^{\mathrm{K}}\langle P\rangle}{1+c_{2}^{\mathrm{K}}\langle P\rangle}$

with

$$
\begin{aligned}
& D_{\mathrm{go}}^{\mathrm{K}}=\frac{2}{3} r_{\mathrm{o}}\left(\frac{8 R T}{\pi M_{\mathrm{g}}}\right)^{1 / 2} \\
& c_{1}^{\mathrm{K}}=2.00\left(\frac{8}{\pi}\right)^{1 / 2} \frac{r_{\mathrm{o}}}{\eta \bar{c}} \\
& c_{2}^{\mathrm{K}}=2.47\left(\frac{8}{\pi}\right)^{1 / 2} \frac{r_{\mathrm{o}}}{\eta \bar{c}}
\end{aligned}
$$

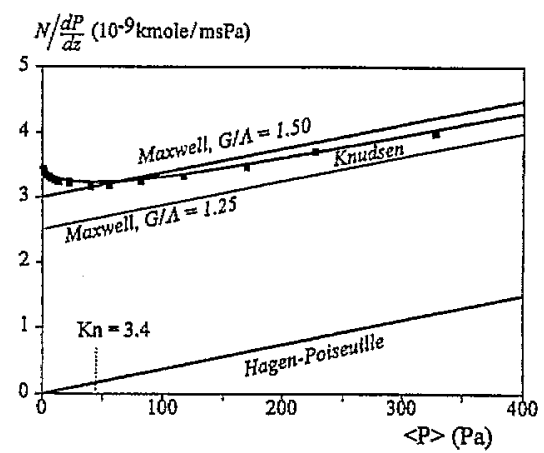

Fig. 8. Data of Knudsen on the flow of $\mathrm{CO}_{2}$ through a capillary; his correlation (Eq. (155) and Eq. (156)) and lines representing Maxwell slip (Eq. (153)) and Hagen-Poiseuille flow. Data taken from Cunningham and Williams [31].

in which $\bar{c}$ is the average molecular speed.

In Fig. 8, a plot of the experimental data is given, together with the results according to the Knudsen correlation, the Hagen-Poiseuille laminar flow equation and the Maxwell slip equation for $G / \Lambda=1.25$ and $G / \Lambda=1.50$. Although we could use the full Knudsen correlation, for engineering purposes we take the linear approximation for $G / \Lambda=1.50$ as being sufficiently accurate. In their modelling of the transport of gases through porous plugs, Mason and Malinauskas [8] also used a constant value of $D^{\mathrm{K}}$. Through the simplified kinetic gas theory equations, the Maxwell slip equation and the linearized Knudsen equation are equivalent; with our choice of $G / \Lambda=1.50$, this corresponds to

$D^{\mathrm{K}} \approx 0.89 D_{\mathrm{o}}^{\mathrm{K}}$

To couple the friction coefficients for pure components to the Knudsen and permeability coefficients, we write the friction equations for a single component

$\frac{1}{P_{\mathrm{g}}} \vec{\nabla} P_{\mathrm{g}}=-r_{\mathrm{gm}} \vec{N}_{\mathrm{g}}$

and thus we have

$\frac{1}{r_{\mathrm{gm}}}=\frac{P_{\mathrm{g}}}{R T}\left(D_{\mathrm{g}}^{\mathrm{K}}+\frac{B_{\mathrm{o}} P_{\mathrm{g}}}{\eta}\right)$

If we use the $\beta$ definition

$\beta_{\mathrm{gm}} \vec{u}_{\mathrm{g}}=r_{\mathrm{gm}} \vec{N}_{\mathrm{g}}$

we have for $\beta$

$\frac{1}{\beta_{\mathrm{gm}}}=\left(D_{\mathrm{g}}^{\mathrm{K}}+\frac{B_{\mathrm{o}} P_{\mathrm{g}}}{\eta}\right)$

which may be formally written as

$\frac{1}{\beta_{\mathrm{gm}}}=\frac{1}{\beta_{\mathrm{gm}}^{\mathrm{K}}}+\frac{1}{\beta_{\mathrm{gm}}^{\mathrm{v}}}$

\subsubsection{Gas mixture permeation}

For the Knudsen flow of a mixture, we have 
$\frac{1}{P_{\mathrm{t}}} \vec{\nabla} P_{i}=-\frac{R T}{D_{i}^{\mathrm{K}}} \frac{\vec{N}_{i}}{P_{\mathrm{t}}}$

from which follows

$\beta_{i \mathrm{~m}}^{\mathrm{K}}=\frac{x_{i}}{D_{i}^{\mathrm{K}}}$

since the interspecies friction term is absent in the Knudsen region.

For the normal region, we consider again the hypothetical HVMF situation: for all components, the local and poreaveraged velocities are equal. For this system, we stated that for all intents and purposes it can be regarded as purely viscous flow, given by Darcy's law, which may be written as

$\frac{1}{P_{\mathrm{t}}} \vec{\nabla} P_{\mathrm{t}, \mathrm{HVMF}}=-\frac{N_{\mathrm{t}} R T}{P^{2}} \frac{\eta}{B_{\mathrm{o}}}$

and from Eq. (152), and by using the $\beta$ notation

$\frac{1}{P_{\mathrm{t}}} \vec{\nabla} P_{\mathrm{t}, \mathrm{HVMF}}=-\sum_{i=1}^{n} \beta_{i \mathrm{~m}}^{\mathrm{v}} \vec{u}_{i}=-\vec{u} \sum_{i=1}^{n} \beta_{i \mathrm{~m}}^{\mathrm{v}}=-\frac{N_{\mathrm{t}} R T}{P} \sum_{i=1}^{n} \beta_{i \mathrm{~m}}^{\mathrm{v}}$

Now, as before, we write

$\beta_{i \mathrm{~m}}^{v}=\frac{1}{B_{0}} \kappa_{i} \phi_{i}=\frac{1}{B_{0}} \kappa_{i} x_{i}$

and so

$\sum_{i=1}^{n} \kappa_{i} x_{i}=\frac{\eta}{P_{\mathrm{t}}}$

If we knew the relation between the viscosity and composition, we could evaluate $\kappa$. From the Chapman-Enskog theory, it follows [39]

$\eta=\sum_{i=1}^{n} \frac{x_{i} \eta_{i}^{o}}{\sum_{j=1}^{n} x_{j} \xi_{i j}}$

in which $\xi_{i j}$ are the interaction parameters and $\eta_{i}^{\circ}$ are the pure component viscosities. This gives for $\kappa$

$\kappa_{i}=\frac{1}{P_{\mathrm{t}}} \frac{\eta_{i}^{\circ}}{\sum_{j=1}^{n} x_{j} \xi_{i j}}$

and for $\beta$

$\beta_{i \mathrm{~m}}^{\mathrm{v}}=\frac{1}{B_{\circ} P_{\mathrm{t}}} \frac{\eta_{i}^{\circ} x_{i}}{\sum_{j=1}^{n} x_{j} \xi_{i j}}$

We now assume that we may find the component-wall friction factors for a mixture by means of the same addition as for the single gas flow

$\beta_{i \mathrm{~m}}=\left(\frac{D_{i}^{\mathrm{K}}}{x_{i}}+\frac{1}{\beta_{i \mathrm{~m}}^{\mathrm{v}}}\right)^{-1}=x_{i}\left(D_{i}^{\mathrm{K}}+\frac{B_{0}}{\kappa_{i}}\right)^{-1}$
For convenience's sake, we introduce a modified coefficient

$f_{i \mathrm{~m}}=\beta_{i \mathrm{~m}} / x_{i}$

and so

$f_{i \mathrm{~m}}=\left(D_{i}^{\mathrm{K}}+\frac{B_{0}}{\kappa_{i}}\right)^{-1}$

The fundamental problem remains of what to do with the intermolecular momentum exchange in the transition region. At this point, we formally introduce a transition function which is assumed to be equal to zero in the Knudsen regime and unity in the fully normal regime, and introduce it into the Maxwell-Stefan term

$\vec{\nabla}_{T, P} x_{i}+\frac{x_{i}}{P_{\mathrm{t}}} \vec{\nabla}_{T} P_{\mathrm{t}}=R T \sum_{j=1}^{n} \Phi_{i j} \frac{\left(x_{i} \vec{N}_{j}-x_{j} \vec{N}_{i}\right)}{P_{\mathrm{t}} D_{i j}}-f_{i \mathrm{~m}} \frac{R T}{P_{\mathrm{t}}} \vec{N}_{i}$

We assume that we can retain symmetry by writing the formal relationship

$\Phi_{i j}=\Phi_{i j}\left(\theta_{i j}\right)$

$\theta_{i j}=\frac{\Lambda_{i j}}{r_{\mathrm{o}}}$

in which $\Lambda$ is some averaged mean free path

$\Lambda_{i j}=\frac{R T}{\sqrt{2} \pi \sigma_{\mathrm{c} i P}^{2} P_{\mathrm{t}} N_{\mathrm{av}}}$

and $\sigma_{c i j}$ is the collision diameter.

There are several ways to order the Maxwell-Stefan equation (Eq. (176)). Here we choose partial pressures rather than mole fractions. Multiplying both sides of Eq. (176) by $P_{\mathrm{t}}$ we obtain

$\vec{\nabla}_{T} P_{i}=R T \sum_{j=1}^{n} \Phi_{i j} \frac{\left(P_{i} \vec{N}_{j}-P_{j} \vec{N}_{i}\right)}{P_{i} \bigoplus_{i j}}-f_{i \mathrm{~m}} R T \vec{N}_{i}$

which can be written in matrix form as

$(\nabla P)=[\mathbf{A}](N)$

with

$[\mathbf{A}]_{i j}=R T\left(P_{i} \frac{\Phi_{i j}}{P_{\mathrm{t}} D_{i j}}-\delta_{i j} \sum_{k=1}^{n} \frac{\Phi_{i k} P_{k}}{P_{\mathrm{t}} D_{i k}}\right)-\delta_{i j} f_{i \mathrm{~m}} R T$

For a binary system, we obtain

$\nabla P_{1}=R T \frac{\Phi_{12}}{P_{1} \bigoplus_{12}}\left(P_{1} N_{2}-P_{2} N_{1}\right)-f_{1 \mathrm{~m}} R T N_{1}$

$\nabla P_{2}=R T \frac{\Phi_{12}}{P_{\mathrm{t}} D_{12}}\left(P_{2} N_{1}-P_{1} N_{2}\right)-f_{2 \mathrm{~m}} R T N_{2}$

\subsection{Permeation of gases through porous plugs}

In many papers, the results of Evans et al. $[28,29]$ on the permeation of $\mathrm{He}$ and $\mathrm{Ar}$ through a low permeability porous graphite septum have been used to illustrate the validity of 


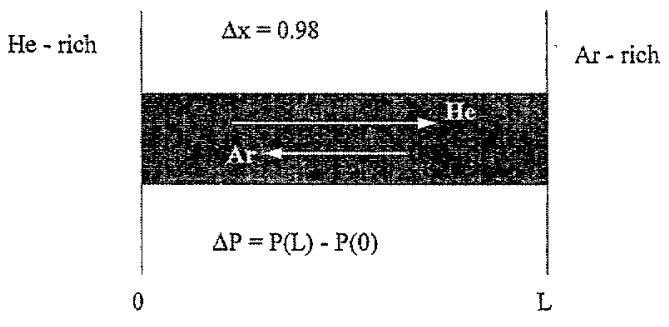

pemeation through graphite septum (Evans et al, 1962)

Fig. 9. Schematic set-up of experiments of Evans et al. [28,29].

the DGM, e.g. Refs. $[8,13]$. It was therefore of interest to investigate the predictions made with the BFM and compare the results with those from the DGM. We assume that there is no mass transfer resistance other than that inside the septum. The experimental set-up is sketched schematically in Fig. 9. We wish to find the steady state fluxes for a porous membrane, at both sides of which the partial pressures of the components are fixed. First, we convert Eq. (180) to equations including porosity and tortuosity effects

$(\nabla P)=\left[\mathbf{A}^{\prime}\right]\left(N^{\prime}\right)$

$\left[\mathbf{A}^{\prime}\right]_{i j}=[\mathbf{A}]_{i j} \frac{\tau}{\epsilon}$

If we had the exact flux values, simultaneous integration of Eq. (183) over the membrane thickness by means of a suitable simultaneous initial value integrator would give the correct values of the partial pressures at the end of the pore, $(P)^{\mathrm{L}}$. We define a target function vector

$(g)=(P)_{\text {calc }}^{\mathrm{L}}-(P)_{\text {obs }}^{\mathrm{L}}$

which we want to approach the zero vector.

We have guessed values $\left(N^{\prime}\right)$, with which we calculate $g$ and write

$(g)=\left(g^{\prime}\right)+[\mathbf{J}]\left(N^{\prime}-N^{\prime}\right)$

in which the elements of the Jacobian matrix are given by

$$
[\mathbf{J}]_{i j}=\left(\frac{\partial g_{i}}{\partial N^{\prime}}\right)
$$

Thus a new estimate for the fluxes is

$$
\left(N^{\prime}\right)=\left(N^{\prime}\right)-[\mathbf{J}]^{-1}\left(g^{\cdot}\right)
$$

which may be modified by means of a suitable relaxation vector

$$
\left(N^{\prime}\right)=\left(N^{\prime \cdot}\right)-[\mathbf{r}][\mathbf{J}]^{-1}(g)
$$

in which $[\mathbf{r}]$ has only diagonal elements.

We may distinguish between two types of system. In one type, there is a constant system pressure, and as a consequence $P_{t}$ is constant. In this case, only $n-1$ fluxes and partial pressures should be considered, and that of $n$ is trivial. In another set-up, a total pressure difference is present over the membrane, and the system is solved for the $n$ components, evaluating $P_{\mathrm{t}}$ at each location by means of summing over the components.

For a binary gas mixture, we have the modified equations

$$
\begin{aligned}
& \nabla P_{1}=\frac{\tau}{\epsilon}\left[R T \frac{\Phi_{12}}{P_{1} \oplus_{12}}\left(P_{1} N^{\prime}{ }_{2}-P_{2} N^{\prime}{ }_{1}\right)-f_{1 \mathrm{~m}} R T N^{\prime}{ }_{1}\right] \\
& \nabla P_{2}=\frac{\tau}{\epsilon}\left[R T \frac{\Phi_{12}}{P_{\mathrm{t}} \oplus_{12}}\left(P_{2} N^{\prime}{ }_{1}-P_{1} N^{\prime}{ }_{2}\right)-f_{2 \mathrm{~m}} R T N^{\prime}{ }_{2}\right]
\end{aligned}
$$

In one of their experimental sets, Evans et al, maintained a zero pressure difference over the septum. For this specific situation, we can eliminate the flux of component 2 , and derive

$N_{2}=-N_{1} \frac{f_{1 \mathrm{~m}}}{f_{2 \mathrm{~m}}}$

$\nabla P_{1}=\frac{\tau}{\epsilon} R T\left[\frac{\Phi_{12}}{P_{\mathrm{t}} D_{12}}\left(P_{1}\left(1-\frac{f_{1 \mathrm{~m}}}{f_{2 \mathrm{~m}}}\right)-P_{\mathrm{t}}\right)-f_{\mathrm{lm}}\right] N^{\prime}{ }_{1}$

and the fluxes can be obtained by direct numerical integration of Eq. (191)

$$
\begin{aligned}
& N^{\prime}{ }_{1}=\frac{\epsilon}{\tau} \frac{1}{R T L_{\mathrm{m}}} \int_{P_{1}(0)}^{P_{1}\left(L_{\mathrm{m})}\right)} \frac{\mathrm{d} P_{1}}{\frac{\Phi_{12}}{P_{\mathrm{t}} \Xi_{12}}\left(P_{1}\left(1-\frac{f_{1 \mathrm{~m}}}{f_{2 \mathrm{~m}}}\right)-P_{\mathrm{t}}\right)-f_{1 \mathrm{~m}}} \\
& N^{\prime}{ }_{2}=\frac{\epsilon}{\tau} \frac{1}{R T L_{\mathrm{m}}} \int_{P_{1}(0)}^{P_{1}\left(L_{\mathrm{m})}\right)} \frac{f_{1 \mathrm{~m}}}{f_{2 \mathrm{~m}}} \frac{\mathrm{d} P_{1}}{\frac{\Phi_{12}}{P_{\mathrm{t}} D_{12}}\left(P_{1}\left(1-\frac{f_{1 \mathrm{~m}}}{f_{2 \mathrm{~m}}}\right)-P_{\mathrm{t}}\right)-f_{1 \mathrm{~m}}}
\end{aligned}
$$

For the molecular diffusion coefficient, we used the value of $7.29 \times 10^{-5} \mathrm{~m}^{2} \mathrm{~s}^{-1}$ at 1 bar, as given by Reid et al. [39], assuming inverse proportionality with pressure. For the permeability $B_{0}$, we used a value of $1.66 \times 10^{-14} \mathrm{~m}^{2}$, and for the correction factor, a value of $\epsilon / \tau$ of $1.28 \times 10^{-4}$, which is slightly different from that of Evans et al. The Knudsen coefficients for $\mathrm{He}$ and $\mathrm{Ar}$ were taken as $3.07 \times 10^{-4}$ and $9.72 \times 10^{-5} \mathrm{~m}^{2} \mathrm{~s}^{-1}$ respectively, corresponding to the effective values of Mason and Malinauskas [8]. For the viscosity and fractional viscosity coefficients, Eq. (170) and Eq. (171) were used, with $\xi_{i j}$ according to Wilke [39]. We assumed for a first approximation $\Phi_{12} \approx 1$, and in Fig. 10 the results are shown. We see that the coverage is good, except at higher pressures, in which the He flow predicted by the model is lower than the experimental values. The Ar flux is predicted well over the whole range. It should be noted that similar agreement and discrepancies were shown by Krishna [13] for the DGM.

In other experiments, Evans et al. varied the pressure drop over the septum at various levels of the average total pressure and constant compositions at both sides. For a first estimate, we linearized Eq. (183) by taking the pressure gradients as the ratio of the pressure drop over the membrane thickness, and by evaluating the coefficients $A$ at the average pressure 


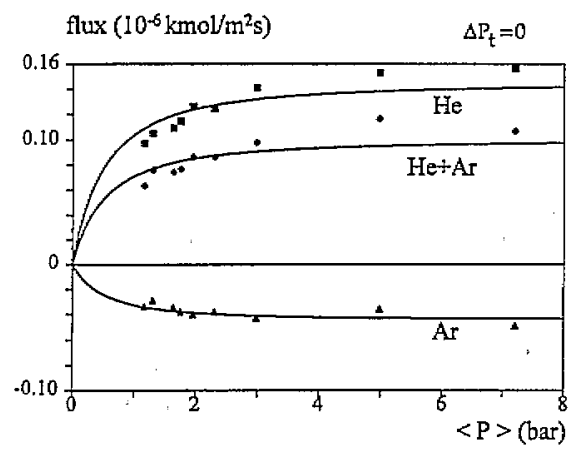

Fig. 10. Fluxes of He and Ar through a low permeability graphite septum in the case of zero total pressure difference over the septum at variable average pressure. Composition difference $\Delta x_{\mathrm{He}}=0.963$. Symbols, experimental data of Evans et al. [28]; lines, according to binary friction model.

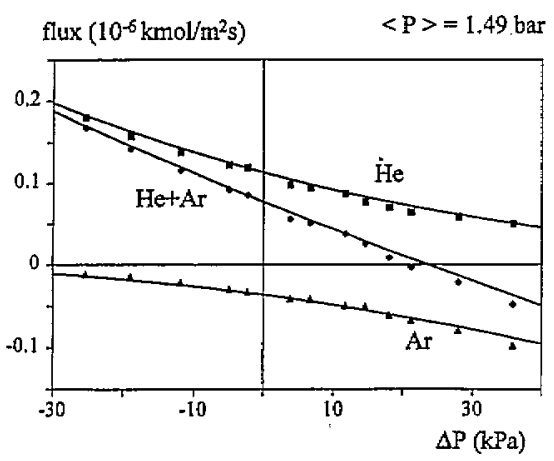

Fig. 11. Fluxes of He and Ar through a low permeability graphite septum in the case of a constant average pressure of 1.49 bar at varying pressure difference over the septum. Composition difference $\Delta x_{\mathrm{He}}=0.963$. Symbols, experimental data of Evans et al. [29]; lines, according to binary friction model.

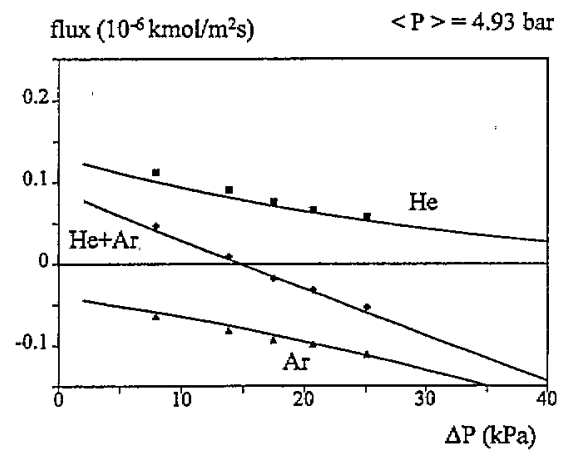

Fig. 12. Same as in Fig. 11, but with an average total pressure of 4.93 bar.

and composition, according to the linearized method of Krishna [13]. Subsequently, we solved the equations for the fluxes by means of the Crout algorithm [40]. We took these values as starting values for the numerical integration and iteration procedure; convergence was reached in all cases after five iterations. This showed that, in all cases, the Ar fluxes only deviated a few per cent from the linearized estimate; however, the He flux was adjusted by about $10 \%-20 \%$.

In Fig. 11 and Fig. 12 some results are shown. We see that, for the average pressure of $1.49 \mathrm{bar}$, the model describes the data well, and only shows minor deviations for the Ar flux at large pressure differences. At the 4.93 bar level, we obtain

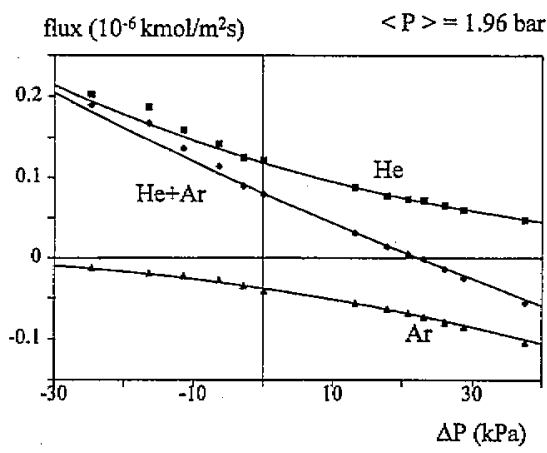

Fig. 13. Same as in Fig. 11, but with an average total pressure of 1.96 bar.

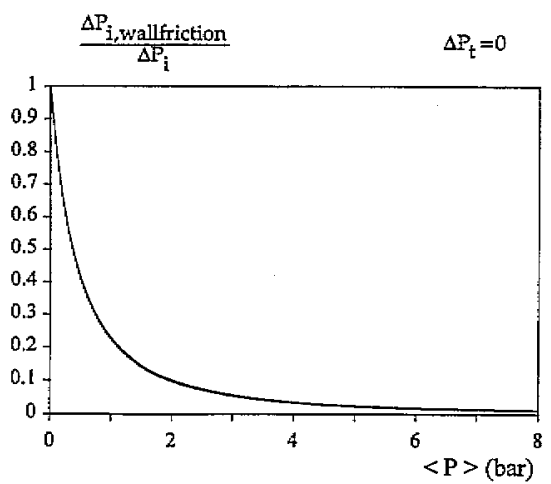

Fig. 14. Relative contribution of the wall friction to the total friction for both $\mathrm{He}$ and $\mathrm{Ar}$ in the case of an equal pressure experiment, according to the binary friction model.

excellent correspondence. In Fig. 13, we show the data at $1.96 \mathrm{bar}$; we see a small deviation in the He flux at strongly negative pressure differences. The same result was found for the DGM by Krishna [13], although the correspondence of the present model is slightly better. Mason and Malinauskas [8] found a slightly better coverage of the experimental data.

It is of interest to determine why both the DGM and BFM give approximately the same results, since the difference lies in the contribution of the wall friction. In Fig. 14, the relative contribution of the wall friction of a component to its total pressure drop is plotted, for the case of equal pressure permeation, as follows from the present model. The curves of both components coincide, and approach unity at zero pressure, as follows from the fact that in the Knudsen region only wall friction occurs. For higher pressures, the interspecies momentum exchange takes over an increasing part of the partial pressure differences, leaving only a minor contribution for the wall friction. In Fig. 15, the relative wall friction is plotted for the case of net pressure drop across the septum. We see that, again, with increasing total pressure, the contribution of the wall friction decreases for both components. In addition, the effect of wall friction is highest for the component which has to move against the total pressure drop; this effect is somewhat stronger for Ar than for He, as may be expected because the pure Ar viscosity is higher and, consequently, so is its $\kappa$ value.

At very low pressures the DGM predicts Knudsen flow, as does the BFM, and so in this region the results correspond. 


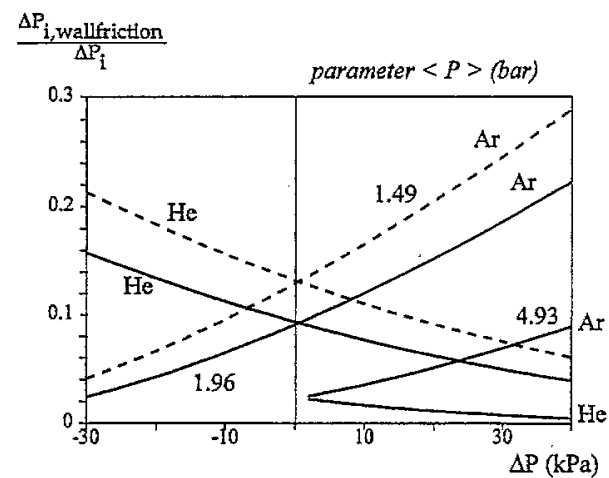

Fig. 15. Same as in Fig. 14, but for the case of a constant average total pressure and varying pressure drop.

At pressures of 2 bar and higher, the average contribution of the wall friction is below $20 \%$ of the total friction; a deviation between the models of $25 \%$ will still make a difference of only $5 \%$ or less in the total friction forces, or in the fluxes at given pressure gradients.

\subsection{Slip phenomena in capillary transport}

Here we present some of the results of the BFM for gas counterdiffusion in capillaries. In view of the already considerable length of this paper, the discussion is limited to a number of examples without an in-depth evaluation of the physical phenomena themselves. A more complete treatment will be reserved for future work. Kramers and Kistemaker [33] were the first to consider and measure the so-called "diffusive slip", later also called the "baro-effect". They measured the interdiffusion of $\mathrm{N}_{2}$ and $\mathrm{H}_{2}$ in a capillary of 0.4 $\mathrm{m}$ length and $0.92 \mathrm{~mm}$ diameter at $18{ }^{\circ} \mathrm{C}$, the ends of which were connected to two large reservoirs, initially filled with pure gases. The reservoirs were separated by a rubber membrane, and from the displacement of a needle glued to the membrane the pressure difference was measured. After some time a quasi-steady state develops in which there is no net molar flux and the pressure difference no longer changes; for finite reservoirs this is inevitably followed by a decreasing pressure difference towards equilibrium. In Fig. 16, the results of the BFM according to Eq. (192) are plotted. A qualitative agreement with the results of Kramers and Kistemaker [33] is obtained; at lower average pressures, the pressure difference increases.

Waldmann and Schmitt [41] made similar measurements for a broader range of gas pairs, and observed the pressure difference in time between two reservoirs of 64.2 and 32.1 $\mathrm{cm}^{3}$ connected by a capillary of $0.048 \mathrm{~m}$ length and 0.207 $\mathrm{mm}$ diameter at $20^{\circ} \mathrm{C}$. The reservoirs formed an integral part with a manometer system filled with silicon oil, with which the pressure differences were measured. As in the experiments of Kramers and Kistemaker [33], the pressure difference builds up, but then reaches a maximum, after which the system goes slowly to equilibrium. In view of the solubility of ethylene and $\mathrm{CO}_{2}$, it was remarked that the experimental accuracy for these gases is low. In Fig. 17, we compare the

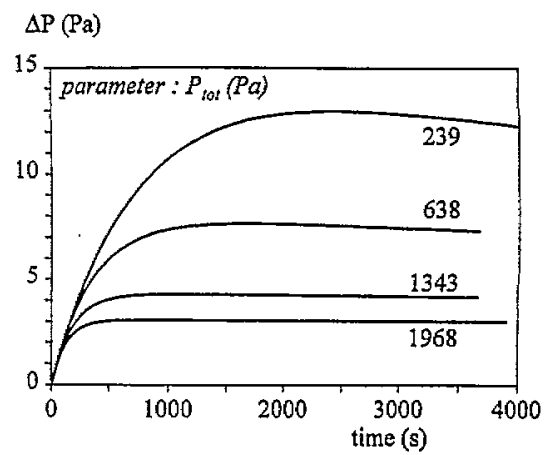

Fig. 16. Simulated results with the binary friction model for the counterdiffusion of $\mathrm{H}_{2}$ and $\mathrm{N}_{2}$ in the Kramers and Kistemaker experiments [33].

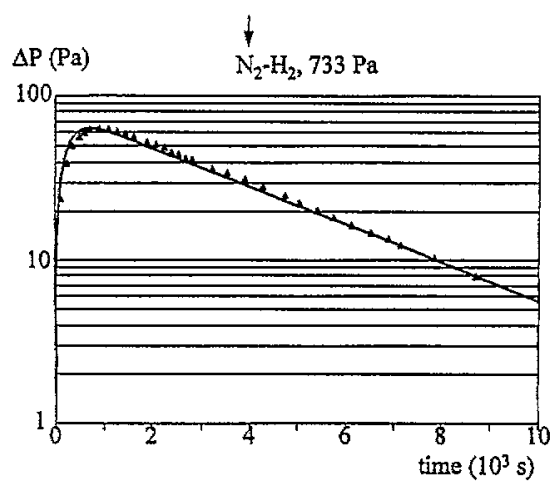

Fig. 17. Net pressure difference in the counterdiffusion of $\mathrm{N}_{2}$ and $\mathrm{H}_{2}$. Symbols, experimental data of Waldmann and Schmitt [41]; drawn line, simulation with the binary friction model.

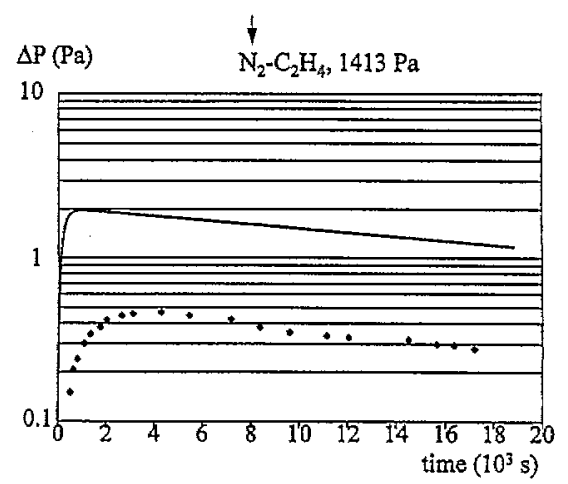

Fig. 18. Net pressure difference in the counterdiffusion of $\mathrm{N}_{2}$ and $\mathrm{C}_{2} \mathrm{H}_{4}$ Symbols, experimental data of Waldmann and Schmitt [41]; drawn line, simulation with the binary friction model. Highest pressure on the nitrogen side.

results of the BFM with the experimental data for one of the measurements with the system $\mathrm{N}_{2}-\mathrm{H}_{2}$, and observe excellent agreement. As observed by Lang and Loyalka [42], the theoretical approximation of Kramers and Kistemaker [33] is not valid for components with equal masses; it predicts a zero pressure difference in contrast with the experimental findings of Waldmann and Schmitt [41] for the system $\mathrm{N}_{2}-\mathrm{C}_{2} \mathrm{H}_{4}$, who observed a net pressure difference. In Fig. 18, we again compare the BFM results with the experimental data and see that the BFM is in agreement and describes a net pressure drop for gases of equal molar mass. In experiments with Ar- 


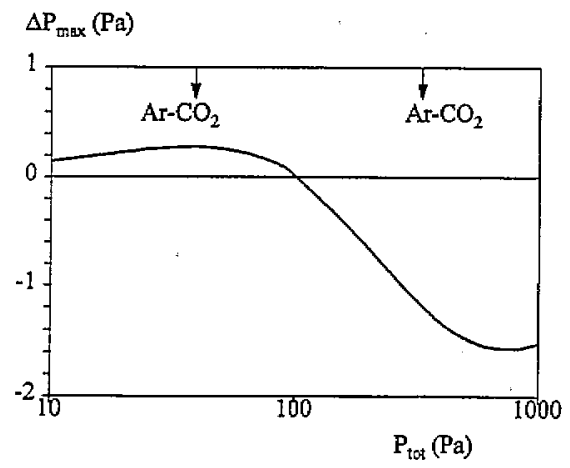

Fig. 19. Reversal of the maximum pressure difference in the counterdiffusion of $\mathrm{Ar}$ and $\mathrm{CO}_{2}$ as simulated with the binary friction model for the experimental situation of Waldmann and Schmitt [41]. At low average pressure, highest pressures on the $\mathrm{CO}_{2}$ side.

$\mathrm{CO}_{2}$, Waldmann and Schmitt [41] observed that, at low average pressures, the maximum pressure occurs at the $\mathrm{CO}_{2}$ side, as expected from the molecular masses, but at higher pressures this effect is reversed. In Fig. 19, the results of the $\mathrm{BFM}$ are shown, illustrating this behaviour. Again, because of the solubility of $\mathrm{CO}_{2}$ in the manometer oil, the experimental accuracy is low [41], and no exact comparison can be made. It should be noted that the last two effects could not be described by the DGM [30].

In all the calculations, the physical data were calculated according to the correlations and physical data presented by Reid et al. [39], and the coefficient $\Phi_{i j}$ was taken to be equal to unity.

\section{Concluding remarks}

The BFM as derived here has been shown to give good agreement with the ultrafiltration results for a single case. The model has the practical advantage that the friction parameters with the membrane follow from free solution viscosity measurements, and so in addition to the free solution parameters only the distribution coefficient between the free solution and the membrane remains as an adjustable parameter. It is of great interest to investigate whether the application for other systems is successful, especially for multicomponent systems, and we intend to do so in our laboratory. One interesting aspect is the fact that, for PEG-3400, the solution diffusion coefficient inside the pore can be taken to be equal to that in free solution. For larger molecules, we may expect that this approach should break down towards a ratio of $1 / 3$ because of the loss of two degrees of spatial freedom. However, it may also be expected that this will have only a small effect on the retention and transmembrane pressure drop, since the equilibrium distribution coefficient will drop to such low values that this will be the determining factor.

In a fundamental sense, the boundary layer model offers a better perspective for the calculation of thermodynamic interactions of components combined with turbulent and molecular diffusion.
The recognition of the errors in the derivations of the DGM will hopefully lead to an end of the long-time discussion about the differences between the Lightfoot and the Mason approach. It also means that the basic picture of parallel viscous and diffusive flow is a distortion of the definition of fluxes with respect to the mass-averaged velocity. It would be better to replace it with the additivity of average interspecies and species-wall friction forces.

The BFM has also been shown to work for binary gas transport in pores, with species-wall friction coefficients from mixture viscosity data and effective Knudsen coefficients. It will be interesting to test the model for ternary gas systems.

It has been shown that the BFM correctly predicts diffusive slip as well as viscous slip phenomena. One of the interesting studies to be undertaken involves the correspondence and discrepancies between the rather simple expressions in the $\mathrm{BFM}$ and the statistical mechanical results.

From an engineering viewpoint, it is felt that the BFM provides a higher accessibility to multicomponent transport than previous models, since the friction coefficients can now be estimated from basic data.

\section{Acknowledgements}

The author gratefully acknowledges the discussions with Carien van Oers, Marius Vorstman and Frank Pijpers, which led to the search for alternatives of the theory. In the final stages, many discussions with Krzysztof Ptasinski were very helpful. The financial support of the Innovatiegericht Onderzoek Programma Membranen, Stichting Toegepaste Wetenschappen, AKZO-NOBEL, Gist-brocades, Shell/KSLA, Stork-Friesland, Unilever Research and X-Flow has been of great importance for our membrane research programme. Enthusiastic discussions with representatives of these programmes and companies have provided a strong driving force for the search for a clear picture of the phenomena in ultrafiltration.

The clarity of treatment in the book of Taylor and Krishna [23] led the way to the translation of ideas into the correct mathematical framework.

The reviewers of previous versions of the manuscript pointed out an erroneous interpretation of the wall friction forces and insufficient account for slip flow, which enabled the appropriate expressions to be found.

\section{Appendix A. Nomenclature}

[A] friction matrix for gases (see Eq. (180))

[B] Maxwell-Stefan friction matrix $\left(\mathrm{s} \mathrm{m}^{-2}\right)$

$B_{0} \quad$ permeability parameter $\left(\mathrm{m}^{2}\right)$

$\tilde{B}_{j} \quad$ See Eq. (55)

$c$ molar concentration $\left(\mathrm{kmol} \mathrm{m}^{-3}\right)$

$\bar{c} \quad$ average molecular speed $\left(\mathrm{m} \mathrm{s}^{-1}\right)$ 


\begin{tabular}{|c|c|}
\hline$c_{1}^{\mathrm{K}}, c_{2}^{\mathrm{K}}$ & Knudsen constants ( see Eq. (156)) $\left(\mathrm{Pa}^{-1}\right)$ \\
\hline $\begin{array}{l}c_{\text {Vieth }} \\
\nexists\end{array}$ & $\begin{array}{l}\text { constant (see Eq. (116)) } \\
\text { Maxwell-Stefan diffusion coefficient }\left(\mathrm{m}^{2} \mathrm{~s}^{-1}\right)\end{array}$ \\
\hline$D$ & Fickian diffusion coefficient $\left(\mathrm{m}^{2} \mathrm{~s}^{-1}\right)$ \\
\hline$D_{i}^{\mathrm{K}}$ & $\begin{array}{l}\text { Knudsen diffusion coefficient of component } i \\
\left(\mathrm{~m}^{2} \mathrm{~s}^{-1}\right)\end{array}$ \\
\hline$D_{i 0}^{\mathrm{K}}$ & $\begin{array}{l}\text { Knudsen diffusion coefficient of component } i \text { at } \\
\text { zero pressure limit }\left(\mathrm{m}^{2} \mathrm{~s}^{-1}\right)\end{array}$ \\
\hline $\bar{D}_{\mathrm{m}}, \bar{D}_{\mathrm{m}}^{\circ}$ & $\begin{array}{l}\text { averaged Mason diffusion coefficients (see } \\
\text { Eq. (28) and Eq. (29)) }\left(\mathrm{m}^{2} \mathrm{~s}^{-1}\right)\end{array}$ \\
\hline$D^{\prime}{ }_{i j}$ & $\begin{array}{l}\text { diffusion coefficient including second-order } \\
\text { corrections (see Eq. }(20))\left(\mathrm{m}^{2} \mathrm{~s}^{-1}\right)\end{array}$ \\
\hline$d$ & diameter $(\mathrm{m})$ \\
\hline$\vec{d}$ & $\begin{array}{l}\text { generalized driving force in Lightfoot } \\
\text { derivation }\left(\mathrm{m}^{-1}\right)\end{array}$ \\
\hline$E_{i j}$ & augmented diffusion coefficients $\left(\mathrm{m}^{2} \mathrm{~s}^{-1}\right)$ \\
\hline$\vec{e}$ & $\begin{array}{l}\text { generalized driving force in present derivation } \\
\left(\mathrm{m}^{-1}\right)\end{array}$ \\
\hline$\tilde{F}$ & external force per unit mass $\left(\mathrm{N} \mathrm{kg}^{-1}\right)$ \\
\hline$\tilde{F}^{\prime}$ & zero vector used in Lightfoot derivation (see \\
\hline
\end{tabular}

\section{Eq. (3))}

$\tilde{F}_{i}$ external force per molar mass unit $\left(\mathrm{N} \mathrm{kmol}^{-1}\right)$ matrix in convective part of transport equation ( $\mathrm{s} \mathrm{m}^{-2}$ )

Fanning friction factor

fraction of molecules diffusively reflected from wall

modified wall friction factor (see Eq. (174))

Maxwell slip modulus ( $\mathrm{m}$ )

generalized friction matrix $\left(\mathrm{s} \mathrm{m}^{-2}\right)$

target function vector (see Eq. (184))

gravitation vector $\left(\mathrm{m} \mathrm{s}^{-2}\right)$

friction coefficient (see Eq. (68))

identity matrix

component

Jacobian matrix (see Eq. (186))

flux with respect to mass-averaged velocity $(\mathrm{kg}$ $\mathrm{m}^{-2} \mathrm{~s}^{-1}$ )

equilibrium constant

convective friction matrix (see Eq. (131))

mass transfer coefficient $\left(\mathrm{m} \mathrm{s}^{-1}\right)$

length (m)

dimensionless mixing length

molecular mass $\left(\mathrm{kg} \mathrm{kmol}^{-1}\right)$

flux with respect to stationary coordinate $(\mathrm{kmol}$ $\mathrm{m}^{-2} \mathrm{~s}^{-1}$ )

number of components

constant in Deissler equation (Eq. (117))

pressure $(\mathrm{Pa})$

gas constant $\left(\mathrm{J} \mathrm{kmol}^{-1} \mathrm{~K}^{-1}\right)$

pore radius $(\mathrm{m})$

diagonal matrix of relaxation factors

apparent retention (rejection) $\left(1-c_{1 \mathrm{p}} / c_{1 \mathrm{~b}}\right)$

Reynolds number $\left(\left(\rho\left\langle u_{\mathrm{t}}\right\rangle d\right) / \eta\right)$

membrane resistance $\left(\mathrm{m}^{-1}\right)$

modified Reynolds number (see Eq. (110))
Fickian matrix inside membrane (see

Eq. $(131))\left(\mathrm{m}^{2} \mathrm{~s}^{-1}\right)$

Schmidt number $(\eta /(\rho D))$

Sherwood number $((k d) / D)$

Stanton number $\left(k /\left\langle u_{\mathrm{t}}\right\rangle\right)$

absolute temperature $(\mathrm{K})$

linear velocity of component $i\left(\mathrm{~m} \mathrm{~s}^{-1}\right)$

modified velocity (see Eq. (110)) $\left(\mathrm{m} \mathrm{s}^{-1}\right)$

dimensionless velocity (see Eq. (110))

bulk velocity along membrane $\left(\mathrm{m} \mathrm{s}^{-1}\right)$

specific molar volume $\left(\mathrm{m}^{3} \mathrm{kmol}^{-1}\right)$

mass-averaged velocity $\left(\mathrm{m} \mathrm{s}^{-1}\right)$

second-order viscosity correction (see

Eq. (34)) (Pa s)

mole fraction

distance from surface in boundary layer $\left(d_{\text {tubc }} /\right.$ $2-z)(\mathrm{m})$

distance coordinate $(\mathrm{m})$

Bearman force per mole (see Eq. (54)) (N

$\mathrm{kmol}^{-1}$ )

\section{A.1. Greek letters}

$\alpha^{\prime}{ }_{i} \quad$ selectivity coefficient of Mason and del Castillo [2]

$\alpha_{\mathrm{m}} \quad$ membrane resistance factor $\left(1 / B_{0}\right)\left(\mathrm{m}^{-2}\right)$

$\beta$ friction coefficient $\left(\mathrm{s} \mathrm{m}^{-2}\right)$

$\gamma \quad$ activity coefficient

$\Gamma \quad$ thermodynamic factor

$\delta \quad$ Kronecker delta

$\delta_{\text {pol }} \quad$ thickness of polarization (boundary) layer

$\epsilon$ porosity

$\eta \quad$ dynamic viscosity (Pa $\mathrm{s}$ )

$\eta_{i} \quad$ fractional viscosity of component $i$ ( $\mathrm{Pa} \mathrm{s}$ )

$\eta_{i}^{\circ} \quad$ viscosity of pure component $i$ (Pas)

$\theta_{i j}$

$\lambda$

$\Lambda$

$\mu$

$\nu$

$\xi_{i j}$

$\Pi$

$\rho$

$\sigma$

$\sigma$

$\sigma_{i j}$

s

$\tau$

$\phi$

$\phi$

$\Phi_{i j}$

$\omega$

$\Omega$ averaged ratio of mean free path and pore radius

mixing length parameter

mean free path $(\mathrm{m})$

chemical potential $\left(\mathrm{J} \mathrm{kmol}^{-1}\right)$

kinematic viscosity $\left(\mathrm{m}^{2} \mathrm{~s}^{-1}\right)$

interaction parameters in Eq. (170)

osmotic pressure $(\mathrm{Pa})$

mass concentration $\left(\mathrm{kg} \mathrm{m}^{-3}\right)$

entropy production ( $\mathrm{W} \mathrm{m}^{-3} \mathrm{~K}^{-1}$ )

osmotic reflection coefficient

collision diameter $(\mathrm{m})$

viscosity parameter (see Eq. (55))

tortuosity

volume fraction

fractional viscosity parameter ( $s$ )

correction factor for diffusion in transition

region (see Eq. (176))

weight fraction $\left(\mathrm{kg} \mathrm{kg}^{-1}\right)$

collision integral (see Eq. (53)) 


\section{A.2. Vector, tensor notation}

$\vec{\nabla}, \nabla \quad$ gradient operator, in porous system along pore path $\left(\mathrm{m}^{-1}\right)$

$\vec{u} \quad$ pore-averaged velocity along pore path

$\tilde{u} \quad$ velocity with three-dimensional components

- inner product notation

[] square matrix

() component vector

\section{A.3. Subscripts}

a air

act actual

app apparent

b bulk

c concentration base

d dust

flow available for flow

g gas

$i, j \quad$ component

int intrinsic

$\mathrm{m}$ membrane, per unit membrane area

m molecular

n number of components

$n$ number-averaged

permeate

pore pore

$\mathrm{s} \quad$ solvent

sp specific

$t$ total

tot total

turb turbulent

visc viscous

w water

\section{A.4. Superscripts}

D diffusive

K Knudsen

$\mathrm{m}$ per unit area of membrane

pore (see Eq. (74))

thermal

viscous, in $\vec{N}_{i}^{\mathrm{y}}$

volume-averaged, in $u^{\mathrm{v}}$

dimensionless boundary layer quantity

infinite pore size

inside liquid phase in membrane

estimated value

$$
\begin{aligned}
\frac{c_{i} \bar{V}_{i}}{R T} \nabla P & =-\frac{c_{i} \bar{V}_{i}}{R T}\left(\frac{c_{\mathrm{t}} R T}{B_{\mathrm{o}}} \sum_{j=1}^{n} \kappa_{j} \phi_{j} u_{j}\right) \\
& =-\frac{c_{i} \bar{V}_{i} c_{\mathrm{t}}}{B_{\mathrm{o}}} \sum_{j=1}^{n} \kappa_{j} N_{j} \bar{V}_{j} \\
& =-\frac{c_{i} \bar{V}_{i} c_{\mathrm{t}}}{B_{\mathrm{o}}}\left(\sum_{j=1}^{n-1} \kappa_{j} N_{j} \bar{V}_{j}+\kappa_{n} N_{n} \bar{V}_{n}\right) \\
& =-\frac{c_{i} \bar{V}_{i} c_{\mathrm{t}}}{B_{\mathrm{o}}}\left(\sum_{j=1}^{n-1} \kappa_{j} N_{j} \bar{V}_{j}+\kappa_{n} \bar{V}_{n}\left(N_{\mathrm{t}}-\sum_{j=1}^{n-1} N_{j}\right)\right) \\
& =-\frac{c_{i} \bar{V}_{i} c_{\mathrm{t}}}{B_{\mathrm{o}}}\left(\sum_{j=1}^{n-1} N_{j}\left(\kappa_{j} \bar{V}_{j}-\kappa_{n} \bar{V}_{n}\right)+\kappa_{n} \bar{V}_{n} N_{\mathrm{t}}\right)
\end{aligned}
$$

The Maxwell-Stefan diffusion term is developed along the lines of Taylor and Krishna [23], resulting in

$$
\sum_{j=1}^{n} \frac{x_{i} N_{j}-x_{j} N_{i}}{D_{i j}}=N_{t} \frac{x_{i}}{\Xi_{i n}}+x_{i} \sum_{j=1}^{n-1} N_{j}\left(\frac{1}{\Xi_{i j}}-\frac{1}{D_{i n}}\right)-N_{i} \sum_{k=1}^{n} \frac{x_{k}}{\bigoplus_{i k}}
$$

The last term in Eq. (78) is written as

$\frac{c_{\mathrm{t}}}{B_{\mathrm{o}}} \kappa_{i} \phi_{i} u_{i}=\frac{c_{\mathrm{t}}}{B_{\mathrm{o}}} \kappa_{i} c_{i} \bar{V}_{i} \frac{N_{i}}{c_{i}}=\frac{c_{\mathrm{t}}}{B_{\mathrm{o}}} \kappa_{i} N_{i} \bar{V}_{i}$

and regrouping the various terms of Eq. (78) gives

$$
\begin{aligned}
c_{\mathrm{t}} \sum_{j=1}^{n-1} \Gamma_{i j} \nabla x_{j}-N_{\mathrm{e}} x_{i}\left(\frac{1}{D_{i n}}+\frac{c_{\mathrm{t}}^{2} \bar{V}_{i} \bar{V}_{n}}{B_{\mathrm{o}}} \kappa_{n}\right) \\
=\frac{c_{\mathrm{t}}^{2} x_{i} \bar{V}_{i}^{n-1}}{B_{\mathrm{o}}} \sum_{j=1}^{n} N_{j}\left(\kappa_{j} \bar{V}_{j}-\kappa_{n} \bar{V}_{n}\right)+x_{i}^{n-1} \sum_{j=1} N_{j}\left(\frac{1}{D_{i j}}-\frac{1}{D_{i n}}\right) \\
\quad-N_{i} \sum_{k=1}^{n} \frac{x_{k}}{D_{i k}}-\frac{c_{\mathrm{t}}}{B_{\mathrm{o}}} \kappa_{i} \bar{V}_{i} N_{i}
\end{aligned}
$$

From this equation, the coefficients given in Eqs. (82)-(85) are obtained.

\section{Appendix C. Relation between $[\mathrm{F}]^{\circ}$ and $[\mathrm{B}](x)$}

\section{We regard the sum}

$$
S_{i}=\sum_{j=1}^{n-1} B_{i j} x_{j}
$$

The second term of Eq. (78) can be written with Eq. (73) as

\section{Appendix B. Derivation of the flux equations for the binary friction model}

Substitution of $B_{i j}$ gives 


$$
\begin{aligned}
& S_{i}=\sum_{j=1}^{n-1} B_{i j} x_{j} \\
& =\sum_{j=1}^{n-1} B_{i j} x_{j}+B_{i j} x_{i} \\
& =-\sum_{\substack{j=1 \\
j \neq i}}^{n-1} x_{i} x_{j}\left(\frac{1}{\Xi_{i j}}-\frac{1}{D_{i n}}\right)+\frac{x_{i}^{2}}{D_{i n}}+\sum_{\substack{j=1 \\
k \neq i}}^{n-1} x_{k}\left(\frac{1}{D_{i k}}\right) \\
& =-\sum_{\substack{j=1 \\
j \neq i}}^{n-1} x_{i} x_{j}\left(\frac{1}{\Phi_{i j}}-\frac{1}{\Xi_{i n}}\right)+\frac{x_{i}^{2}}{\Xi_{i n}}+\sum_{\substack{j=1 \\
k \neq i}}^{n-1} x_{k}\left(\frac{1}{D_{i k}}\right) \\
& =-x_{i}\left\{\sum_{j=1}^{n} x_{j}\left(\frac{1}{D_{i j}}\right)-\frac{x_{n}}{D_{i n}}\right\}+\frac{x_{i}}{D_{i n}}\left(1-x_{i}-x_{n}\right)+\frac{x_{i}^{2}}{D_{i n}} \\
& +\sum_{j=1}^{n-1} x_{k}\left(\frac{1}{D_{i k}}\right) \\
& =\frac{x_{i}}{D_{i n}}
\end{aligned}
$$

and so we have

$[\mathbf{F}]^{\infty}(x)=[\mathbf{B}](x)$

\section{Appendix D. Derivations in connection with concentration basis}

\section{D.1. $\Gamma$ factor}

We have

$$
\begin{aligned}
c_{\mathrm{t}} \frac{x_{i}}{R T} \nabla_{T, P} \mu_{i} & =\frac{c_{i}}{R T} \nabla_{T, P} \mu_{i}=\frac{c_{i}}{R T} \sum_{j=1}^{n-1} \frac{\partial\left(R T \ln a_{i}\right)}{\partial c_{j}} \nabla_{T, P} c_{j} \\
& =c_{i} \sum_{j=1}^{n-1} \frac{\partial\left(\ln a_{i}\right)}{\partial c_{j}} \nabla_{T, p} c_{j}
\end{aligned}
$$

For the activity, we may write

$\ln a_{i}=\ln \gamma_{i}+\ln c_{i}-\ln c_{t}$

and so

$$
\begin{aligned}
\frac{\partial\left(\ln a_{i}\right)}{\partial c_{j}} & =\frac{\partial\left(\ln \gamma_{i}\right)}{\partial c_{j}}+\frac{\partial\left(\ln c_{i}\right)}{\partial c_{j}}-\frac{\partial\left(\ln c_{t}\right)}{\partial c_{j}} \\
& =\frac{\partial\left(\ln \gamma_{i}\right)}{\partial c_{j}}+\delta_{i j}-\frac{\partial\left(\ln c_{i}\right)}{\partial c_{j}}
\end{aligned}
$$

For the total concentration, we may write

$$
c_{\mathrm{t}}=\sum_{j=1}^{n-1} c_{j}+c_{n}=\sum_{j=1}^{n-1} c_{j}+\left(1-\sum_{j=1}^{n-1} c_{j} \bar{V}_{j}\right) / \bar{V}_{n}
$$

and thus

$$
\frac{\partial\left(c_{\mathrm{t}}\right)}{\partial c_{j}}=1-\frac{\bar{V}_{j}}{\bar{V}_{n}}
$$

leading to the result

$$
c_{\mathrm{t}} \frac{x_{i}}{R T} \nabla_{T, P} \mu_{i}=\sum_{j=1}^{n-1} \Gamma_{\mathrm{c}, i j} \nabla c_{j}
$$

in which $\Gamma_{\mathrm{c}}$ is given by

$$
\left[\Gamma_{\mathrm{c}}\right]_{i j}=\Gamma_{c, i j}=\delta_{i j}+c_{i} \frac{\partial \ln \gamma_{i}}{\partial c_{j}}-\frac{c_{i}}{c_{\mathrm{t}}}\left(1-\frac{\vec{V}_{j}}{\overline{\bar{V}}}\right)
$$

\section{D.2. The diffusion term}

We split up the term with the Maxwell-Stefan equations into

$$
\begin{aligned}
c_{t} \sum_{j=1}^{n} \frac{x_{i} N_{j}-x_{j} N_{i}}{D_{i j}} & =\sum_{j=1}^{n} \frac{c_{i} N_{j}-c_{j} N_{i}}{D_{i j}} \\
& =\sum_{j=1}^{n-1} \frac{c_{i} N_{j}-c_{j} N_{i}}{D_{i j}}+\frac{c_{i} N_{n}-c_{n} N_{i}}{D_{i n}}
\end{aligned}
$$

and with

$$
\begin{aligned}
& N_{n}=N_{\mathrm{t}}-\sum_{j=1}^{n-1} N_{j} \\
& c_{n}=c_{\mathrm{t}}-\sum_{j=1}^{n-1} c_{j}
\end{aligned}
$$

we have for the last term

$\frac{c_{i} N_{n}-c_{n} N_{i}}{\bigoplus_{i n}}=\frac{c_{i}}{\Xi_{i n}}\left(N_{t}-\sum_{j=1}^{n-1} N_{j}\right)-\frac{N_{i}}{D_{i n}} c_{n}$

leading to

$$
\begin{gathered}
c_{\mathrm{t}} \sum_{j=1}^{n} \frac{x_{i} N_{j}-x_{j} N_{i}}{\Xi_{i j}}=N_{\mathrm{t}} \frac{c_{i}}{\Xi_{i n}}+c_{i} \sum_{j=1}^{n-1} \frac{N_{j}}{\bigoplus_{i j}}-N_{i} \sum_{j=1}^{n-1} \frac{c_{j}}{D_{i j}}-\frac{c_{i}}{\bigoplus_{i n}} \sum_{j=1}^{n-1} N_{j} \\
-N_{i} \frac{c_{n}}{\Xi_{i n}}=N_{i} \frac{c_{i}}{\Xi_{i n}}+c_{i} \sum_{j=1}^{n-1} \frac{N_{j}}{D_{i j}}-N_{i} \sum_{j=1}^{n} \frac{c_{j}}{\bigoplus_{i j}}-\frac{c_{i}}{\Xi_{i n}} \sum_{j=1}^{n-1} N_{j}
\end{gathered}
$$

and so the coefficients defined by

$c_{\mathrm{t}} \sum_{j=1}^{n} \frac{x_{i} N_{j}-x_{j} N_{i}}{D_{i j}}=-[\mathbf{B}]_{\mathrm{c}}(N)$

are given by

$$
\begin{aligned}
& {[\mathbf{B}]_{\mathrm{c}, i i}=\frac{c_{i}}{D_{i n}}+\sum_{\substack{k=1 \\
k \neq i}}^{n} \frac{c_{k}}{D_{i k}}} \\
& {[\mathbf{B}]_{\mathrm{c}, i j}=-c_{i}\left(\frac{1}{D_{i j}}-\frac{1}{D_{i n}}\right) \quad i \neq j}
\end{aligned}
$$

and it is easy to see that $[\mathbf{B}]_{\mathrm{c}}=c_{\mathrm{t}}[\mathbf{B}]$ 


\section{References}

[1] E.N. Lightfoot, Transport in Living Systems, Wiley, New York, 1974.

[2] E.A. Mason and L.F. del Castillo, J. Membr. Sci., 23 (1985) 199.

[3] E.A. Mason and L.A. Viehland, J. Chem. Phys., 68 (1978) 3562.

[4] G.D. Mehta, T.F. Morse, E.A. Mason and M.H. Daneshpajooh, J. Chem. Phys, 64 (1976) 3917.

[5] L.F. del Castillo, E.A. Mason and H.E. Revercomb, Biophys. Chem., IO (1979) 191.

[6] L.F. del Castillo and E.A. Mason, Biophys. Chem., 12 (1980) 223.

[7] M.H. Daneshpajooh, E.A. Mason, E.H. Bresler and R.P. Wendt, Biophys. J., 15 (1975) 591.

[8] E.A. Mason and A.P. Malinauskas, Gas Transport in Porous Media: The Dusty-Gas Model, Elsevier, Amsterdam, 1983.

[9] E.A. Mason and H.K. Lonsdale, J. Membr. Sci., 51 (1990) 1.

[10] K.S. Spiegler, IEC Fundam., 5 (1966) 529.

[11] B.C. Robertson and A.L. Zydney, Sep. Sci. Technol., 23 (1988) 1799.

[12] R. Krishna, Chem. Eng. Commun., 59 (1987) 33.

[13] R. Krishna, Chem. Eng. J., 35 (1987) 75.

[14] P. Vonk, Diffusion of large molecules in porous structures, $P h . D$. Thesis, University of Groningen, 1994.

[15] C.W. van Oers, Solute rejection in multicomponent systems during ultrafiltration, Ph.D. Thesis, Eindhoven University of Technology, 1994.

[16] C.W. van Oers, M.A.G. Vorstman and P.J.A.M. Kerkhof, J. Membr. Sci., 73 (1995) 231.

[17] C.W. van Oers, M.A.G. Vorstman and P.J.A.M. Kerkhof, Preprints Annual Meeting AIChE, 1992, AIChE, 1992, p. 802.

[18] P.J.A.M. Kerkhof and G.H. Schoutens, in S. Bruin (ed.), Preconcentration and Drying of Food Materials, Elsevier, Amsterdam, 1988, p. 87.

[19] P.J.A.M. Kerkhof, Modelling of some problems in industrial enzyme ultrafiltration, Workshop on Recent Advances in Bio Process Technology, Graz, 14-16 March, 1988.

[20] P.J.A.M. Kerkhof, Some problems in membrane processing of enzymes, Biotech '88, Wembley, London, 10-12 May, 1988.

[21] J.L. Anderson and J.A. Quinn, Biophys. J., 14 (1974) 130.
[22] P.M. Bungay and H. Brenner, Int. J. Multiphase Flow, I (1973) 25.

[23] R. Taylor and R. Krishna, Multicomponent Mass Transfer, Wiley, New York, 1993.

[24] G.D.C. Kuiken, Thermodynamics of Ireversible Processes, Wiley, Chichester, 1994.

[25] J.A. Wesselingh and R. Krishna, Mass Transfer, Ellis Horwood, New York, 1990.

[26] R.J. Bearman and J.G. Kirkwood, J. Chem. Phys., 28 (1958) 136.

[27] R.J. Bearman, J. Chem. Phys., 31 (1959) 751.

[28] R.B. Evans, G.M. Watson and J. Truitt, J. Appl. Phys., 33 (1962) 2682.

[29] R.B. Evans, G.M. Watson and J. Truitt, J. Appl. Phys., 34 (1963) 2020.

[30] E.A. Mason, A.P. Malinauskas and R.B. Evans III, J. Chem. Phys., 46 (8) (1967) 3199.

[31] R.E. Cunningham and R.J.J. Williams, Diffision in Gases and Porous Media, Plenum, New York, 1980.

[32] R. Krishna, Chem. Eng. J., 24 (1982) 163.

[33] H.A. Kramers and J. Kistemaker, Physica X, 8 (1943) 699.

[34] R.B. Bird, W.E. Stewart and E.N. Lightfoot, Transport Phenomena, Wiley, New York, 1960.

[35] J.C. Maxwell, Scientific Papers, Vol. II, p. 86; in the edition by W.D. Niven, Dover Publications, New York, 1965, p. 681.

[36] R. Krishna, Chem. Eng. Sci., 48 (1993) 845.

[37] E.N. Sieder and G.E. Tate, Ind. Eng. Chem., 28 (1936) 1429.

[38] K. Meeuwsen, Invloed van een Aerosil-gellaag op de retentie van polyethyleen-glycol bij ultrafiltratie, Ir. Thesis, Eindhoven University of Technology, 1993.

[39] R.C. Reid, J.M. Prausnitz and T.K. Sherwood, The Properties of Gases and Liquids, McGraw-Hill, New York, 1977.

[40] W.H. Press, B.P. Flannery, S.A. Teukolsky and W.T. Vetterling, Numerical Recipes in Pascal, Cambridge University Press, 1989.

[41] L. Waldmann and K.H. Schmitt, Z. Naturforsch., Teil A, 16 (1961) 1343.

[42] H. Lang and S.K. Loyalka, Phys. Fluids, 13 (1970) 1871. 